\title{
Aissa Guesmia \\ The effect of the heat conduction of types I and III on the decay rate of the Bresse system via the longitudinal displacement
}

Received: 14 November 2017 / Accepted: 15 May 2018 / Published online: 14 June 2018 (C) The Author(s) 2018

\begin{abstract}
In this paper, we consider the thermoelastic Bresse system in one-dimensional bounded interval under mixed homogeneous Dirichlet-Neumann boundary conditions and two different kinds of dissipation working only on the longitudinal displacement and given by heat conduction of types I and III. We prove that the exponential stability of the two systems is equivalent to the equality of the three speeds of the wave propagations. Moreover, when at least two speeds of the wave propagations are different, we show the polynomial stability for each system with a decay rate depending on the smoothness of the initial data. The results of this paper complete the ones of Afilal et al. [On the uniform stability for a linear thermoelastic Bresse system with second sound (submitted), 2018], where the dissipation is given by a linear frictional damping or by the heat conduction of second sound. The proof of our results is based on the semigroup theory and a combination of the energy method and the frequency domain approach.
\end{abstract}

Mathematics Subject Classification $35 \mathrm{~B} 40 \cdot 35 \mathrm{~L} 45 \cdot 74 \mathrm{H} 40 \cdot 93 \mathrm{D} 20 \cdot 93 \mathrm{D} 15$

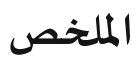

في هذا البحث، نعتبر نظام بريس حراري في مجال محدود في بعد واحد وذلك تحت شروط ديريشلت-نيومان الحدية المختلطة والمتجانسة وبوجود نوعين من التبديدات يعملان فقط على الإزاحة الطولية والمعطيان بتوصيل حراري من نوع I واIII. نثبت أن الإستفرار الأسي يكافئ تساوي السرعات الثادئ الثاثلة لانتشار الأمواج. بالاضافة، نبيّن أنه في حالة اختلاف سرعتين على الأقل، يكون الاستقرار لكل نطام جبريا (كثيري حدود) وذلك الكات بمعدّل تناقص (خمود) يعتمد على ملوسة المعطيات الابتدائية. إن نتائج هذا البحث تكمّل تلك التي أثبتت في التئي [1]، حيث أن التبديد هناك كان نتيجة تخميد احتكاك خطي أو توصيل حراري من نوع الصوت الهوت الثاني (صيغة كتانيو). يستند برهان نتائجنا على نظرية شبه الزمر مع دمج لطريقة الطاقة ومقاربة المجالات الترديد التردية

A. Guesmia $(\varangle)$

Institut Elie Cartan de Lorraine, UMR 7502, Université de Lorraine, 3 Rue Augustin, Fresnel, BP 45112, 57073 Metz Cedex 03 , France

E-mail: aissa.guesmia@univ-lorraine.fr

A. Guesmia

Department of Mathematics and Statistics, King Fahd University of Petroleum and Minerals, Dhahran 31261, Saudi Arabia 


\section{Introduction}

We study in this paper the asymptotic behavior at infinity of the solutions of two coupled systems related to the Bresse model with two different types of dissipation given by heat conduction and working only on the longitudinal displacement. The first system is the Bresse system with thermoelasticity of type I

$$
\begin{cases}\rho_{1} \varphi_{t t}-k\left(\varphi_{x}+\psi+l w\right)_{x}-l k_{0}\left(w_{x}-l \varphi\right)=0 & \text { in }(0,1) \times(0, \infty), \\ \rho_{2} \psi_{t t}-b \psi_{x x}+k\left(\varphi_{x}+\psi+l w\right)=0 & \text { in }(0,1) \times(0, \infty), \\ \rho_{1} w_{t t}-k_{0}\left(w_{x}-l \varphi\right)_{x}+l k\left(\varphi_{x}+\psi+l w\right)+\delta \theta_{x}=0 & \text { in }(0,1) \times(0, \infty), \\ \rho_{3} \theta_{t}-\beta \theta_{x x}+\delta w_{x t}=0 & \text { in }(0,1) \times(0, \infty)\end{cases}
$$

along with the initial data

$$
\begin{cases}\varphi(x, 0)=\varphi_{0}(x), \varphi_{t}(x, 0)=\varphi_{1}(x) & \text { in }(0,1) \\ \psi(x, 0)=\psi_{0}(x), \psi_{t}(x, 0)=\psi_{1}(x) & \text { in }(0,1) \\ w(x, 0)=w_{0}(x), w_{t}(x, 0)=w_{1}(x) & \text { in }(0,1) \\ \theta(x, 0)=\theta_{0}(x) & \text { in }(0,1)\end{cases}
$$

and the mixed homogeneous Dirichlet-Neumann boundary conditions

$$
\left\{\begin{array}{l}
\varphi(0, t)=\psi_{x}(0, t)=w_{x}(0, t)=\theta(0, t)=0 \text { in }(0, \infty) \\
\varphi_{x}(1, t)=\psi(1, t)=w(1, t)=\theta_{x}(1, t)=0 \text { in }(0, \infty) .
\end{array}\right.
$$

The second system is the Bresse system with thermoelasticity of type III

$$
\begin{cases}\rho_{1} \varphi_{t t}-k\left(\varphi_{x}+\psi+l w\right)_{x}-l k_{0}\left(w_{x}-l \varphi\right)=0 & \text { in }(0,1) \times(0, \infty), \\ \rho_{2} \psi_{t t}-b \psi_{x x}+k\left(\varphi_{x}+\psi+l w\right)=0 & \text { in }(0,1) \times(0, \infty), \\ \rho_{1} w_{t t}-k_{0}\left(w_{x}-l \varphi\right)_{x}+l k\left(\varphi_{x}+\psi+l w\right)+\delta \theta_{x t}=0 & \text { in }(0,1) \times(0, \infty), \\ \rho_{3} \theta_{t t}-\beta \theta_{x x}-\gamma \theta_{x x t}+\delta w_{x t}=0 & \text { in }(0,1) \times(0, \infty)\end{cases}
$$

along with (2) and (3), and

$$
\theta_{t}(x, 0)=\theta_{1}(x) \text { in }(0,1),
$$

where $\rho_{1}, \rho_{2}, \rho_{3}, b, k, k_{0}, \delta, \beta, \gamma$ and $l$ are positive constants, $w, \varphi$ and $\psi$ represent, respectively, the longitudinal, vertical and shear angle displacements, and $\theta$ denotes the temperature.

Several well-posedness and stability results for Bresse systems [2] have been obtained during the last few years, where the stability depends on the nature and position of the controls and some relations between the coefficients. Let us mention here some known results concerning the thermoelastic Bresse systems. For more details in what concerns mathematical modeling of the thermoelastic problems, we refer the readers to the works $[3,6,7,10,11]$.

The authors of [13] considered the following system:

$$
\left\{\begin{array}{l}
\rho_{1} \varphi_{t t}-k\left(\varphi_{x}+\psi+l w\right)_{x}-l k_{0}\left(w_{x}-l \varphi\right)+l \delta \theta=0 \\
\rho_{2} \psi_{t t}-b \psi_{x x}+k\left(\varphi_{x}+\psi+l w\right)+\delta q_{x}=0 \\
\rho_{1} w_{t t}-k_{0}\left(w_{x}-l \varphi\right)_{x}+l k\left(\varphi_{x}+\psi+l w\right)+\delta \theta_{x}=0 \\
\rho_{3} \theta_{t}-\theta_{x x}+\beta\left(w_{x}-l \varphi\right)_{t}=0 \\
\rho_{3} q_{t}-q_{x x}+\beta \psi_{x t}=0
\end{array}\right.
$$


and proved the exponential stability if

$$
k-k_{0}=\rho_{1} b-\rho_{2} k=0,
$$

and the polynomial stability in general. In [5], the authors proved that

$$
\left\{\begin{array}{l}
\rho_{1} \varphi_{t t}-k\left(\varphi_{x}+\psi+l w\right)_{x}-l k_{0}\left(w_{x}-l \varphi\right)=0, \\
\rho_{2} \psi_{t t}-b \psi_{x x}+k\left(\varphi_{x}+\psi+l w\right)+\delta \theta_{x}=0, \\
\rho_{1} w_{t t}-k_{0}\left(w_{x}-l \varphi\right)_{x}+l k\left(\varphi_{x}+\psi+l w\right)=0, \\
\rho_{3} \theta_{t}-\theta_{x x}+\left(\beta \psi_{t}\right)_{x}=0
\end{array}\right.
$$

is exponentially stable if and only if (7) holds, and it is polynomially stable in general. The results of [5] were generalized in [15] to the case where $\delta$ and $\beta$ are functions of $x$ and vanish on some part of the domain. The authors of [9] proved that the following thermoelastic Bresse system

$$
\left\{\begin{array}{l}
\rho_{1} \varphi_{t t}-k\left(\varphi_{x}+\psi+l w\right)_{x}-l k_{0}\left(w_{x}-l \varphi\right)=0 \\
\rho_{2} \psi_{t t}-b \psi_{x x}+k\left(\varphi_{x}+\psi+l w\right)+\delta \theta_{x}=0 \\
\rho_{1} w_{t t}-k_{0}\left(w_{x}-l \varphi\right)_{x}+l k\left(\varphi_{x}+\psi+l w\right)=0 \\
\rho_{3} \theta_{t}+q_{x}+\delta \psi_{x t}=0 \\
\tau q_{t}+\beta q+\theta_{x}=0
\end{array}\right.
$$

is exponentially stable if

$$
k-k_{0}=\left(\frac{\rho_{1}}{k}-\frac{\rho_{2}}{b}\right)\left(1-\frac{\tau k \rho_{3}}{\rho_{1}}\right)-\frac{\tau \delta^{2}}{b}=0 \text { and } l \text { is small, }
$$

it is not exponentially stable if

$$
k \neq k_{0} \quad \text { or } \quad\left(\frac{\rho_{1}}{k}-\frac{\rho_{2}}{b}\right)\left(1-\frac{\tau k \rho_{3}}{\rho_{1}}\right) \neq \frac{\tau \delta^{2}}{b},
$$

and it is polynomially stable in general. The author of [4] studied the stability of

$$
\begin{cases}\rho_{1} \varphi_{t t}-k\left(\varphi_{x}+\psi+l w\right)_{x}-l k_{0}\left(w_{x}-l \varphi\right)=0 & \text { in }(0,1) \times(0, \infty), \\ \rho_{2} \psi_{t t}-b \psi_{x x}+k\left(\varphi_{x}+\psi+l w\right)+\delta \theta_{x}=0 & \text { in }(0,1) \times(0, \infty), \\ \rho_{1} w_{t t}-k_{0}\left(w_{x}-l \varphi\right)_{x}+l k\left(\varphi_{x}+\psi+l w\right)=0 & \text { in }(0,1) \times(0, \infty), \\ \rho_{3} \theta_{t}-\beta \int_{0}^{\infty} g(s) \theta_{x x}(t-s) d s+\delta \psi_{x t}=0 & \text { in }(0,1) \times(0, \infty),\end{cases}
$$

where $g: \mathbb{R}_{+} \rightarrow \mathbb{R}_{+}$is a given function satisfying some hypotheses. He provided a necessary and sufficient condition for exponential stability in terms of the structural parameters of the problem. For particular choices of $g$, the results of [4] cover the cases of Fourier, Cattaneo and Coleman-Gurtin heat conduction.

For all the above stability results, at least the shear angle displacement $\psi$ was damped via the heat conduction. The authors of [1] considered the Cattaneo heat conduction working only on the longitudinal displacement

$$
\left\{\begin{array}{l}
\rho_{1} \varphi_{t t}-k\left(\varphi_{x}+\psi+l w\right)_{x}-l k_{0}\left(w_{x}-l \varphi\right)=0 \\
\rho_{2} \psi_{t t}-b \psi_{x x}+k\left(\varphi_{x}+\psi+l w\right)=0 \\
\rho_{1} w_{t t}-k_{0}\left(w_{x}-l \varphi\right)_{x}+l k\left(\varphi_{x}+\psi+l w\right)+\delta \theta_{x}=0 \\
\rho_{3} \theta_{t}+q_{x}+\delta w_{x t}=0 \\
\tau q_{t}+\beta q+\theta_{x}=0
\end{array}\right.
$$


and proved that the exponential stability is equivalent to

$$
k \rho_{2}-b \rho_{1}=\left(k-k_{0}\right)\left(\rho_{3}-\frac{\rho_{1}}{\tau k}\right)-\delta^{2}=0
$$

and

$$
l^{2} \neq \frac{k_{0} \rho_{2}+b \rho_{1}}{k_{0} \rho_{2}}\left(\frac{\pi}{2}+m \pi\right)^{2}+\frac{k \rho_{1}}{\rho_{2}\left(k+k_{0}\right)}, \quad \forall m \in \mathbb{Z} .
$$

Moreover, the polynomial stability of (10) in general was also proved in [1]. Similar stability results were proved in [1] when $\delta \theta_{x}$ is replaced by $\delta w_{t}$, the last two equations in (10) are neglected and (11) is replaced by (7).

Our objective in this paper is to complete the results of [1] by considering the heat conduction of types I and III. We prove that, when $l$ does not belong to two sequences of real numbers (conditions (15) and (24) below), the exponential stability of the two systems is equivalent to (7). Moreover, we show that the polynomial stability holds in general with two decay rates corresponding to the two cases,

$$
\rho_{1} b-\rho_{2} k=0 \text { and } \rho_{1} b-\rho_{2} k \neq 0 .
$$

The proof of the well-posedness is based on the semigroup theory. However, the stability results are proved using the energy method combined with the frequency domain approach.

The paper is organized as follows. In Sect. 2, we give an idea on the proof of the well-posedness of (1)-(3) and (2)-(5). In Sects. 3 and 4, we prove, respectively, our exponential and polynomial stability results.

\section{The semigroup setting}

In this section, we give a brief idea on the proof of the well-posedness of (1)-(3) and (2)-(5). We consider the energy space

$$
\mathcal{H}=\tilde{\mathcal{H}} \times \begin{cases}L^{2}(0,1) & \text { in case }(1), \\ H_{*}^{1}(0,1) \times L^{2}(0,1) & \text { in case (4), }\end{cases}
$$

where

$$
\begin{gathered}
\tilde{\mathcal{H}}=H_{*}^{1}(0,1) \times L^{2}(0,1) \times \widetilde{H_{*}^{1}}(0,1) \times{L^{2}}^{2}(0,1) \times \widetilde{H_{*}^{1}}(0,1) \times L^{2}(0,1), \\
H_{*}^{1}(0,1)=\left\{f \in H^{1}(0,1): f(0)=0\right\} \text { and } \tilde{H_{*}^{1}}(0,1)=\left\{f \in H^{1}(0,1): f(1)=0\right\} .
\end{gathered}
$$

The space $\mathcal{H}$ is equipped with the inner product

$$
\begin{aligned}
\left\langle\Phi_{1}, \Phi_{2}\right\rangle_{\mathcal{H}}= & k\left\langle\left(\varphi_{1 x}+\psi_{1}+l w_{1}\right),\left(\varphi_{2 x}+\psi_{2}+l w_{2}\right)\right\rangle_{L^{2}(0,1)}+b\left\langle\psi_{1 x}, \psi_{2 x}\right\rangle_{L^{2}(0,1)} \\
& +k_{0}\left\langle\left(w_{1 x}-l \varphi_{1}\right),\left(w_{2 x}-l \varphi_{2}\right)\right\rangle_{L^{2}(0,1)}+\rho_{1}\left\langle\tilde{\varphi}_{1}, \tilde{\varphi}_{2}\right\rangle_{L^{2}(0,1)}+\rho_{2}\left\langle\tilde{\psi}_{1}, \tilde{\psi}_{2}\right\rangle_{L^{2}(0,1)} \\
& +\rho_{1}\left\langle\tilde{w}_{1}, \tilde{w}_{2}\right\rangle_{L^{2}(0,1)}+\left\{\begin{array}{lc}
\rho_{3}\left\langle\theta_{1}, \theta_{2}\right\rangle_{L^{2}(0,1)} & \text { in case (1), } \\
\beta\left\langle\theta_{1 x}, \theta_{2 x}\right\rangle_{L^{2}(0,1)}+\rho_{3}\left\langle\tilde{\theta}_{1}, \tilde{\theta}_{2}\right\rangle_{L^{2}(0,1)} & \text { in case (4), }
\end{array}\right.
\end{aligned}
$$

where (for $j=1,2$ )

$$
\Phi_{j}= \begin{cases}\left(\varphi_{j}, \tilde{\varphi}_{j}, \psi_{j}, \tilde{\psi}_{j}, w_{j}, \tilde{w}_{j}, \theta_{j}\right)^{T} & \text { in case (1), } \\ \left(\varphi_{j}, \tilde{\varphi}_{j}, \psi_{j}, \tilde{\psi}_{j}, w_{j}, \tilde{w}_{j}, \theta_{j}, \tilde{\theta}_{j}\right)^{T} & \text { in case (4). }\end{cases}
$$

We consider also

$$
\Phi= \begin{cases}(\varphi, \tilde{\varphi}, \psi, \tilde{\psi}, w, \tilde{w}, \theta)^{T} & \text { in case (1), } \\ (\varphi, \tilde{\varphi}, \psi, \tilde{\psi}, w, \tilde{w}, \theta, \tilde{\theta})^{T} & \text { in case (4) }\end{cases}
$$


and

$$
\Phi_{0}= \begin{cases}\left(\varphi_{0}, \varphi_{1}, \psi_{0}, \psi_{1}, w_{0}, w_{1}, \theta_{0}\right)^{T} & \text { in case (1) } \\ \left(\varphi_{0}, \varphi_{1}, \psi_{0}, \psi_{1}, w_{0}, w_{1}, \theta_{0}, \theta_{1}\right)^{T} & \text { in case (4) }\end{cases}
$$

where

$$
\tilde{\varphi}=\varphi_{t}, \quad \tilde{\psi}=\psi_{t}, \quad \tilde{w}=w_{t} \quad \text { and } \quad \tilde{\theta}=\theta_{t} .
$$

Systems (1)-(3) and (2)-(5) can be written as a first-order system given by

$$
\left\{\begin{array}{l}
\Phi_{t}=\mathcal{A} \Phi \quad \text { in }(0, \infty) \\
\Phi(t=0)=\Phi_{0}
\end{array}\right.
$$

where $\mathcal{A}$ is a linear operator defined by

$$
\mathcal{A} \Phi=\left(\begin{array}{c}
\tilde{\varphi} \\
\frac{k}{\rho_{1}}\left(\varphi_{x}+\psi+l w\right)_{x}+\frac{l k_{0}}{\rho_{1}}\left(w_{x}-l \varphi\right) \\
\tilde{\psi} \\
\frac{b}{\rho_{2}} \psi_{x x}-\frac{k}{\rho_{2}}\left(\varphi_{x}+\psi+l w\right) \\
\tilde{w} \\
\frac{k_{0}}{\rho_{1}}\left(w_{x}-l \varphi\right)_{x}-\frac{l k}{\rho_{1}}\left(\varphi_{x}+\psi+l w\right)-\frac{\delta}{\rho_{1}} \theta_{x} \\
\frac{\beta}{\rho_{3}} \theta_{x x}-\frac{\delta}{\rho_{3}} \tilde{w}_{x}
\end{array}\right)
$$

in case (1), and

$$
\mathcal{A} \Phi=\left(\begin{array}{c}
\tilde{\varphi} \\
\frac{k}{\rho_{1}}\left(\varphi_{x}+\psi+l w\right)_{x}+\frac{l k_{0}}{\rho_{1}}\left(w_{x}-l \varphi\right) \\
\tilde{\psi} \\
\frac{b}{\rho_{2}} \psi_{x x}-\frac{k}{\rho_{2}}\left(\varphi_{x}+\psi+l w\right) \\
\tilde{w} \\
\frac{k_{0}}{\rho_{1}}\left(w_{x}-l \varphi\right)_{x}-\frac{l k}{\rho_{1}}\left(\varphi_{x}+\psi+l w\right)-\frac{\delta}{\rho_{1}} \tilde{\theta}_{x} \\
\tilde{\theta} \\
\frac{1}{\rho_{3}}(\beta \theta+\gamma \tilde{\theta})_{x x}-\frac{\delta}{\rho_{3}} \tilde{w}_{x}
\end{array}\right)
$$

in case (4). The domain of $\mathcal{A}$ is defined by

$$
D(\mathcal{A})=\left\{\begin{array}{l}
\Phi \in \mathcal{H} \mid \underset{\sim}{\varphi}, \theta \in H_{*}^{2}(0,1) ; \psi, w \in \tilde{H}_{*}^{2}(0,1) ; \tilde{\varphi} \in H_{*}^{1}(0,1) ; \\
\tilde{\psi}, \tilde{w} \in H_{*}^{1}(0,1) ; \varphi_{x}(1)=\psi_{x}(0)=w_{x}(0)=\theta_{x}(1)=0
\end{array}\right\}
$$

in case (1), and

$$
D(\mathcal{A})=\left\{\begin{array}{l}
\Phi \in \mathcal{H} \mid \underset{\sim}{\varphi}, \beta \theta+\gamma \tilde{\theta} \in H_{*}^{2}(0,1) ; \psi, w \in \widetilde{H_{*}^{2}}(0,1) ; \tilde{\varphi}, \tilde{\theta} \in H_{*}^{1}(0,1) ; \\
\tilde{\psi}, \tilde{w} \in H_{*}^{1}(0,1) ; \varphi_{x}(1)=\psi_{x}(0)=w_{x}(0)=\theta_{x}(1)=0
\end{array}\right\}
$$


in case (4), where

$$
H_{*}^{2}(0,1)=H^{2}(0,1) \cap H_{*}^{1}(0,1) \text { and } \tilde{H_{*}^{2}}(0,1)=H^{2}(0,1) \cap \tilde{H_{*}^{1}}(0,1) .
$$

The following well-posedness results for (14) hold:

Theorem 2.1 Assume that

$$
l \notin \frac{\pi}{2}+\pi \mathbb{N}
$$

Then, for any $m \in \mathbb{N}$ and $\Phi_{0} \in D\left(\mathcal{A}^{m}\right)$, System (14) admits a unique solution

$$
\Phi \in \cap_{j=0}^{m} C^{m-j}\left(\mathbb{R}_{+} ; D\left(\mathcal{A}^{j}\right)\right) .
$$

Proof First, from the definition of $H_{*}^{1}(0,1)$ and $\tilde{H_{*}^{1}}(0,1)$, we see that, if

$$
(\varphi, \psi, w) \in H_{*}^{1}(0,1) \times \widetilde{H_{*}^{1}}(0,1) \times \tilde{H}_{*}^{1}(0,1)
$$

satisfies

$$
k\left\|\left(\varphi_{x}+\psi+l w\right)\right\|_{L^{2}(0,1)}^{2}+b\left\|\psi_{x}\right\|_{L^{2}(0,1)}^{2}+k_{0}\left\|\left(w_{x}-l \varphi\right)\right\|_{L^{2}(0,1)}^{2}=0,
$$

then

$$
\psi=0, \quad \varphi=-c \sin (l x) \text { and } \quad w=c \cos (l x),
$$

where $c$ is a constant such that

$$
c=0 \quad \text { or } l \in \frac{\pi}{2}+\pi \mathbb{N} .
$$

Then Condition (15) implies that $\varphi=\psi=w=0$, and thus, $\mathcal{H}$ is a Hilbert space.

Second, we prove that $\mathcal{A}$ is dissipative. Indeed, using the definition of $\mathcal{A}$ and $\langle\cdot, \cdot\rangle_{\mathcal{H}}$, and integrating by parts, we get

$$
\langle\mathcal{A} \Phi, \Phi\rangle_{\mathcal{H}}= \begin{cases}-\beta\left\|\theta_{x}\right\|_{L^{2}(0,1)}^{2} & \text { in case (1), } \\ -\gamma\left\|\tilde{\theta}_{x}\right\|_{L^{2}(0,1)}^{2} & \text { in case (4). }\end{cases}
$$

Hence, $\mathcal{A}$ is dissipative in $\mathcal{H}$.

Third, we show that, for any $F \in \mathcal{H}$, there exists $Z \in D(\mathcal{A})$ satisfying

$$
\mathcal{A} Z=F,
$$

that is $0 \in \rho(\mathcal{A})$. Let $F=\left(f_{1}, \ldots, f_{j}\right)^{T}$ and $Z=\left(z_{1}, \ldots, z_{j}\right)^{T}$, where $j=7$ in case (1), and $j=8$ in case (4). The first, third and fifth equations in (18) are equivalent to

$$
z_{2}=f_{1}, \quad z_{4}=f_{3} \text { and } z_{6}=f_{5},
$$

and the seventh equation in case (4) becomes

$$
z_{8}=f_{7}
$$

So, because $F \in \mathcal{H}, z_{2}, z_{4}, z_{6}$ and $z_{8}$ have the required regularity in $D(\mathcal{A})$. Then, the last equation in (18) is reduced to

$$
z_{7 x x}=\frac{\delta}{\beta} f_{5 x}+\frac{\rho_{3}}{\beta} f_{7}
$$

in case (1), and

$$
\left(\beta z_{7}+\gamma f_{7}\right)_{x x}=\delta f_{5 x}+\rho_{3} f_{8}
$$

in case (4). By a direct integration, we see that each equation in (21) and (22) has a unique solution $z_{7}$ satisfying the needed regularity and Neumann boundary condition in $D(\mathcal{A})$. Therefore, the second, fourth and sixth equations in (18) become

$$
\left\{\begin{array}{l}
k\left(z_{1 x}+z_{3}+l z_{5}\right)_{x}+l k_{0}\left(z_{5 x}-l z_{1}\right)=\rho_{1} f_{2}, \\
b z_{3 x x}-k\left(z_{1 x}+z_{3}+l z_{5}\right)=\rho_{2} f_{4}, \\
k_{0}\left(z_{5 x}-l z_{1}\right)_{x}-l k\left(z_{1 x}+z_{3}+l z_{5}\right)=\tilde{f},
\end{array}\right.
$$


where

$$
\tilde{f}= \begin{cases}\delta z_{7 x}+\rho_{1} f_{6} & \text { in case (1), } \\ \delta f_{7 x}+\rho_{1} f_{6} & \text { in case (4). }\end{cases}
$$

To prove that (23) admits a solution $\left(z_{1}, z_{3}, z_{5}\right)$ satisfying the required regularity and Neumann boundary condition in $D(\mathcal{A})$, we consider the variational formulation of (23) and use the Lax-Milgram theorem and classical elliptic regularity arguments. So, this proves that (18) has a unique solution $Z \in D(\mathcal{A})$. By the resolvent identity, we have $\lambda I-\mathcal{A}$ is surjective, for any $\lambda>0$ (see [14]). Consequently, the Lumer-Phillips theorem implies that $\mathcal{A}$ is the infinitesimal generator of a linear $C_{0}$ semigroup of contractions on $\mathcal{H}$. Finally, Theorem 2.1 holds (see [16])

\section{Exponential stability}

Our objective in this section is to show the following exponential stability result:

Theorem 3.1 We assume that (15) holds. Then the semigroup associated with (14) is exponentially stable if and only if

$$
l^{2} \neq \frac{\rho_{2} k_{0}+\rho_{1} b}{\rho_{2} k_{0}}\left(\frac{\pi}{2}+m \pi\right)^{2}+\frac{\rho_{1} k}{\rho_{2}\left(k+k_{0}\right)}, \quad \forall m \in \mathbb{Z}
$$

and

$$
k-k_{0}=\rho_{1} b-\rho_{2} k=0 .
$$

The proof is based on the following theorem:

Theorem $3.2[8,17]$ A $C_{0}$ semigroup of contractions on a Hilbert space $\mathcal{H}$ generated by an operator $\mathcal{A}$ is exponentially stable if and only if

$$
i \mathbb{R} \subset \rho(\mathcal{A})
$$

and

$$
\sup _{\lambda \in \mathbb{R}}\left\|(i \lambda I-\mathcal{A})^{-1}\right\|_{\mathcal{L}(\mathcal{H})}<\infty .
$$

Proof We prove that (24) is equivalent to (26), and (25) is equivalent to (27). So Theorem 3.2 implies Theorem 3.1.

\subsection{Conditions (24) and (26) are equivalent}

Note that, according to the fact that $0 \in \rho(\mathcal{A})$ (see Sect. 2), $\mathcal{A}^{-1}$ is bounded and it is a bijection between $\mathcal{H}$ and $D(\mathcal{A})$. Since $D(\mathcal{A})$ has a compact embedding into $\mathcal{H}$, so it follows that $\mathcal{A}^{-1}$ is a compact operator, which implies that the spectrum of $\mathcal{A}$ is discrete. Then $i \lambda \in \rho(\mathcal{A})$ if and only if $\lambda$ is not an eigenvalue of $\mathcal{A}$.

Let $\lambda \in \mathbb{R}^{*}$. We prove that $i \lambda$ is not an eigenvalue of $\mathcal{A}$ by proving that the unique solution $\Phi \in D(\mathcal{A})$ of the equation

$$
\mathcal{A} \Phi=i \lambda \Phi
$$

is $\Phi=0$. Let $\Phi$ be given by (13). The Eq. (28) means that

$$
\left\{\begin{array}{l}
\tilde{\varphi}=i \lambda \varphi, \quad \tilde{\psi}=i \lambda \psi, \quad \tilde{w}=i \lambda w, \\
\frac{k}{\rho_{1}}\left(\varphi_{x}+\psi+l w\right)_{x}+\frac{l k_{0}}{\rho_{1}}\left(w_{x}-l \varphi\right)=i \lambda \tilde{\varphi}, \\
\frac{b}{\rho_{2}} \psi_{x x}-\frac{k}{\rho_{2}}\left(\varphi_{x}+\psi+l w\right)=i \lambda \tilde{\psi}, \\
\frac{k_{0}}{\rho_{1}}\left(w_{x}-l \varphi\right)_{x}-\frac{l k}{\rho_{1}}\left(\varphi_{x}+\psi+l w\right)-\frac{\delta}{\rho_{1}} \theta_{x}=i \lambda \tilde{w}, \\
\frac{\beta}{\rho_{3}} \theta_{x x}-\frac{\delta}{\rho_{3}} \tilde{w}_{x}=i \lambda \theta
\end{array}\right.
$$


in case (1), and

$$
\left\{\begin{array}{l}
\tilde{\varphi}=i \lambda \varphi, \quad \tilde{\psi}=i \lambda \psi, \quad \tilde{w}=i \lambda w, \quad \tilde{\theta}=i \lambda \theta, \\
\frac{k}{\rho_{1}}\left(\varphi_{x}+\psi+l w\right)_{x}+\frac{l k_{0}}{\rho_{1}}\left(w_{x}-l \varphi\right)=i \lambda \tilde{\varphi}, \\
\frac{b}{\rho_{2}} \psi_{x x}-\frac{k}{\rho_{2}}\left(\varphi_{x}+\psi+l w\right)=i \lambda \tilde{\psi}, \\
\frac{k_{0}}{\rho_{1}}\left(w_{x}-l \varphi\right)_{x}-\frac{l k}{\rho_{1}}\left(\varphi_{x}+\psi+l w\right)-\frac{\delta}{\rho_{1}} \tilde{\theta}_{x}=i \lambda \tilde{w}, \\
\frac{1}{\rho_{3}}(\beta \theta+\gamma \tilde{\theta})_{x x}-\frac{\delta}{\rho_{3}} \tilde{w}_{x}=i \lambda \tilde{\theta}
\end{array}\right.
$$

in case (4). Using (17) and (28), we find

$$
0=\operatorname{Re} i \lambda\|\Phi\|_{\mathcal{H}}^{2}=\operatorname{Re}\langle i \lambda \Phi, \Phi\rangle_{\mathcal{H}}=\operatorname{Re}\langle\mathcal{A} \Phi, \Phi\rangle_{\mathcal{H}}= \begin{cases}-\beta\left\|\theta_{x}\right\|_{L^{2}(0,1)}^{2} & \text { in case (1), } \\ -\gamma\left\|\tilde{\theta}_{x}\right\|_{L^{2}(0,1)}^{2} & \text { in case (4). }\end{cases}
$$

Then

$$
\begin{cases}\theta_{x}=0 & \text { in case (1), } \\ \tilde{\theta}_{x}=0 & \text { in case (4). }\end{cases}
$$

But $\theta, \tilde{\theta} \in H_{*}^{1}(0,1)$ (since $\Phi \in D(\mathcal{A})$ ), then, using the Poincaré's inequality, (31) and the fourth equation in (30), we deduce that

$$
\begin{cases}\theta=0 & \text { in case (1), } \\ \theta=\tilde{\theta}=0 & \text { in case (4). }\end{cases}
$$

Therefore, from (32) and the third and last equations in (29) and (30), we find

$$
w_{x}=\tilde{w}_{x}=0 .
$$

As $w, \tilde{w} \in \tilde{H_{*}^{1}}(0,1)$ and according to Poincaré's inequality, we have

$$
w=\tilde{w}=0 .
$$

Using (32) and (34), we see that (29) and (30) are reduced to

$$
\left\{\begin{array}{l}
\tilde{\varphi}=i \lambda \varphi, \quad \tilde{\psi}=i \lambda \psi, \\
\left(l^{2} k_{0}-\rho_{1} \lambda^{2}\right) \varphi-k\left(\varphi_{x}+\psi\right)_{x}=0, \\
-\rho_{2} \lambda^{2} \psi-b \psi_{x x}+k\left(\varphi_{x}+\psi\right)=0, \\
\varphi_{x}+\psi=-\frac{k_{0}}{k} \varphi_{x} .
\end{array}\right.
$$

Now, we follow the proof given in [1]. By deriving the fifth equation in (35) and combining the third one, we see that

$$
\varphi_{x x}+\alpha \varphi=0,
$$

where $\alpha=\frac{l^{2} k_{0}-\rho_{1} \lambda^{2}}{k_{0}}$. We distinguish three cases.

Case $1 \lambda^{2}=\frac{l^{2} k_{0}}{\rho_{1}}$. Then

$$
\varphi(x)=c_{1} x+c_{2},
$$

for $c_{1}, c_{2} \in \mathbb{C}$. Using the boundary conditions

$$
\varphi(0)=\varphi_{x}(1)=0,
$$


we find

$$
\varphi=0,
$$

which implies that, using the first two equations and the last one in (35),

$$
\tilde{\varphi}=0
$$

and

$$
\psi=\widetilde{\psi}=0 .
$$

Consequently, we get

$$
\Phi=0 .
$$

Case $2 \lambda^{2}>\frac{l^{2} k_{0}}{\rho_{1}}$. Then

$$
\varphi(x)=c_{1} e^{\sqrt{-\alpha} x}+c_{2} e^{-\sqrt{-\alpha} x} .
$$

Using again the boundary conditions (37), we find (38), and similarly to case 1, we arrive at (41).

Case $3 \lambda^{2}<\frac{l^{2} k_{0}}{\rho_{1}}$. Then

$$
\varphi(x)=c_{1} \cos (\sqrt{\alpha} x)+c_{2} \sin (\sqrt{\alpha} x) .
$$

Using the boundary conditions (37), we deduce that $c_{1}=0$, and

$$
c_{2}=0 \text { or } \exists m \in \mathbb{Z}: \alpha=\left(\frac{\pi}{2}+m \pi\right)^{2} .
$$

If $c_{2}=0$, then (38) holds, and as before, we find (41).

If $c_{2} \neq 0$, then, by (42), we have

$$
\exists m \in \mathbb{Z}: \frac{l^{2} k_{0}-\rho_{1} \lambda^{2}}{k_{0}}=\left(\frac{\pi}{2}+m \pi\right)^{2} .
$$

Therefore, the fifth equation in (35) is equivalent to

$$
\psi(x)=-c_{2}\left(1+\frac{k_{0}}{k}\right) \sqrt{\alpha} \cos (\sqrt{\alpha} x),
$$

and then the third and fourth equations in (35) are reduced to

$$
\lambda^{2}=\frac{k_{0}\left[k k_{0}+b l^{2}\left(k+k_{0}\right)\right]}{\left(k+k_{0}\right)\left(k_{0} \rho_{2}+b \rho_{1}\right)} .
$$

We see that (43) and (45) lead to

$$
\exists m \in \mathbb{Z}: l^{2}=\frac{\rho_{2} k_{0}+\rho_{1} b}{\rho_{2} k_{0}}\left(\frac{\pi}{2}+m \pi\right)^{2}+\frac{\rho_{1} k}{\rho_{2}\left(k+k_{0}\right)} ;
$$

that is (24) does not hold. So, if (24) holds, we get a contradiction, and hence, $c_{2}=0$ and, as before, we find (41). If (24) does not hold, then, for $\lambda \in \mathbb{R}$ satisfying (45), the function

$$
\begin{aligned}
\Phi(x)= & c_{2}\left(\sin (\sqrt{\alpha} x), i \lambda \sin (\sqrt{\alpha} x),-\left(1+\frac{k_{0}}{k}\right) \sqrt{\alpha} \cos (\sqrt{\alpha} x),\right. \\
& \left.-i \lambda\left(1+\frac{k_{0}}{k}\right) \sqrt{\alpha} \cos (\sqrt{\alpha} x), 0,0,0,0\right)^{T}
\end{aligned}
$$

is a solution of (28), for any $c_{2} \in \mathbb{C}$, and then $\lambda$ is an eigenvalue of $\mathcal{A}$. Finally, (26) holds if and only if (24) holds. 
3.2 Condition (25) implies (27)

We assume that (25) holds and prove (27). Let us proceed by contradiction. So, we assume that (27) is false, then there exist sequences $\left(\Phi_{n}\right)_{n} \subset \mathcal{D}(\mathcal{A})$ and $\left(\lambda_{n}\right)_{n} \subset \mathbb{R}$ satisfying

$$
\begin{aligned}
& \left\|\Phi_{n}\right\|_{\mathcal{H}}=1, \quad \forall n \in \mathbb{N}, \\
& \lim _{n \rightarrow \infty}\left|\lambda_{n}\right|=\infty
\end{aligned}
$$

and

$$
\lim _{n \rightarrow \infty}\left\|\left(i \lambda_{n} I-\mathcal{A}\right) \Phi_{n}\right\|_{\mathcal{H}}=0
$$

\subsubsection{Case of System (1)}

The limit (48) implies the following ones:

$$
\left\{\begin{array}{l}
i \lambda_{n} \varphi_{n}-\tilde{\varphi}_{n} \longrightarrow 0 \text { in } H_{*}^{1}(0,1), \\
i \lambda_{n} \rho_{1} \tilde{\varphi}_{n}-k\left(\varphi_{n x}+\psi_{n}+l w_{n}\right)_{x}-l k_{0}\left(w_{n x}-l \varphi_{n}\right) \longrightarrow 0 \text { in } L^{2}(0,1), \\
i \lambda_{n} \psi_{n}-\tilde{\psi}_{n} \longrightarrow 0 \text { in } \tilde{H}_{*}^{1}(0,1), \\
i \lambda_{n} \rho_{2} \tilde{\psi}_{n}-b \psi_{n x x}+k\left(\varphi_{n x}+\psi_{n}+l w_{n}\right) \longrightarrow 0 \text { in } L^{2}(0,1), \\
i \lambda_{n} w_{n}-\tilde{w}_{n} \longrightarrow 0 \text { in } \tilde{H}_{*}^{1}(0,1) \\
i \lambda_{n} \rho_{1} \tilde{w}_{n}-k_{0}\left(w_{n x}-l \varphi_{n}\right)_{x}+l k\left(\varphi_{n x}+\psi_{n}+l w_{n}\right)+\delta \theta_{n x} \longrightarrow 0 \text { in } L^{2}(0,1), \\
i \lambda_{n} \rho_{3} \theta_{n}-\beta \theta_{n x x}+\delta \tilde{w}_{n x} \longrightarrow 0 \text { in } L^{2}(0,1) .
\end{array}\right.
$$

We will arrive to a contradiction with (46) by proving that

$$
\lim _{n \rightarrow \infty}\left\|\Phi_{n}\right\|_{\mathcal{H}}=0 .
$$

Some of the calculations below are used in [1].

Estimate on $\theta_{n}$ Taking the inner product of $\left(i \lambda_{n} I-\mathcal{A}\right) \Phi_{n}$ with $\Phi_{n}$ in $\mathcal{H}$ and using (17), we get

$$
\operatorname{Re}\left\langle\left(i \lambda_{n} I-\mathcal{A}\right) \Phi_{n}, \Phi_{n}\right\rangle_{\mathcal{H}}=\beta\left\|\theta_{n x}\right\|_{L^{2}(0,1)}^{2} .
$$

Using (46) and (48), we deduce that

$$
\theta_{n x} \longrightarrow 0 \text { in } L^{2}(0,1) \text {. }
$$

Because $\theta_{n}(0)=0$, then we get from (52) that

$$
\theta_{n} \longrightarrow 0 \text { in } L^{2}(0,1) \text {. }
$$

Estimates on $\varphi_{n}, \psi_{n}$ and $w_{n}$ Multiplying (49) $)_{1},(49)_{3}$ and (49) 5 by $\frac{1}{\lambda_{n}}$, and using (46) and (47), we find

$$
\left\{\begin{array}{l}
\varphi_{n} \longrightarrow 0 \text { in } L^{2}(0,1), \\
\psi_{n} \longrightarrow 0 \text { in } L^{2}(0,1), \\
w_{n} \longrightarrow 0 \text { in } L^{2}(0,1)
\end{array}\right.
$$

Estimate on $\frac{1}{\lambda_{n}} w_{n x x}$ Applying the triangle inequality, we have

$$
\begin{aligned}
& \left\|\frac{w_{n x x}}{\lambda_{n}}\right\|\left\|_{L^{2}(0,1)} \leq \frac{1}{k_{0}\left|\lambda_{n}\right|}\right\| i \lambda_{n} \rho_{1} \tilde{w}_{n}-k_{0}\left(w_{n x}-l \varphi_{n}\right)_{x}+l k\left(\varphi_{n x}+\psi_{n}+l w_{n}\right)+\delta \theta_{n x} \|_{L^{2}(0,1)} \\
& +\frac{1}{k_{0}}\left\|i \rho_{1} \tilde{w}_{n}+\frac{l k_{0}}{\lambda_{n}} \varphi_{n x}+\frac{l k}{\lambda_{n}}\left(\varphi_{n x}+\psi_{n}+l w_{n}\right)+\delta \frac{\theta_{n x}}{\lambda_{n}}\right\|_{L^{2}(0,1)} .
\end{aligned}
$$


Then, by (46), (47), (49)6 and (52), we deduce that

$$
\left(\frac{1}{\lambda_{n}} w_{n x x}\right)_{n} \text { is bounded in } L^{2}(0,1) .
$$

Estimates on $w_{n x}, \frac{1}{\lambda_{n}} \widetilde{w}_{n x}$ and $\frac{1}{\lambda_{n}} \widetilde{w}_{n}$ Taking the inner product of $(49)_{7}$ with $\frac{i w_{n x}}{\lambda_{n}}$ in $L^{2}(0,1)$, integrating by parts and using the boundary conditions, we get

$$
\begin{aligned}
& \rho_{3}\left\langle\theta_{n}, w_{n x}\right\rangle_{L^{2}(0,1)}+\beta\left\langle\theta_{n x}, \frac{i w_{n x x}}{\lambda_{n}}\right\rangle_{L^{2}(0,1)}-\delta\left\langle\left(i \lambda_{n} w_{n x}-\tilde{w}_{n x}\right), \frac{i w_{n x}}{\lambda_{n}}\right\rangle_{L^{2}(0,1)} \\
& \quad+\delta\left\|w_{n x}\right\|_{L^{2}(0,1)}^{2} \longrightarrow 0 .
\end{aligned}
$$

Using (46), (47), (49)5, (52), (53) and (55), we deduce that

$$
w_{n x} \longrightarrow 0 \text { in } L^{2}(0,1)
$$

and from $(49)_{5}$, we have

$$
\frac{1}{\lambda_{n}} \widetilde{w}_{n x} \longrightarrow 0 \text { in } L^{2}(0,1)
$$

As $\widetilde{w}_{n}(1)=0$ and using (57), we obtain

$$
\frac{1}{\lambda_{n}} \widetilde{w}_{n} \longrightarrow 0 \text { in } L^{2}(0,1)
$$

Estimates on $\tilde{w}_{n}$ and $\lambda_{n} w_{n}$ Taking the inner product of (49) 6 with $\frac{i \tilde{w}_{n}}{\lambda_{n}}$ in $L^{2}(0,1)$, integrating by parts and using the boundary conditions, we see that

$$
\begin{aligned}
& \rho_{1}\left\|\tilde{w}_{n}\right\|_{L^{2}(0,1)}^{2}+k_{0}\left\langle\left(w_{n x}-l \varphi_{n}\right), \frac{i \tilde{w}_{n x}}{\lambda_{n}}\right\rangle_{L^{2}(0,1)} \\
& +l k\left\langle\left(\varphi_{n x}+\psi_{n}+l w_{n}\right), \frac{i \tilde{w}_{n}}{\lambda_{n}}\right\rangle_{L^{2}(0,1)}+\delta\left\langle\frac{\theta_{n x}}{\lambda_{n}}, i \tilde{w}_{n}\right\rangle_{L^{2}(0,1)} \longrightarrow 0 .
\end{aligned}
$$

Using (46), (47), (52), (57) and (58), we obtain

$$
\widetilde{w}_{n} \longrightarrow 0 \text { in } L^{2}(0,1),
$$

and with (49)5, we find

$$
\lambda_{n} w_{n} \longrightarrow 0 \text { in } L^{2}(0,1) \text {. }
$$

Estimates on $\varphi_{n x}, \tilde{\varphi}_{n}$ and $\lambda_{n} \varphi_{n}$ First, taking the inner product of $\left(\varphi_{n x}+\psi_{n}+l w_{n}\right)$ with $i \lambda_{n} \tilde{w}_{n}$ in $L^{2}(0,1)$, integrating by parts and using the boundary conditions, we have

$$
\begin{aligned}
\left\langle\left(\varphi_{n x}+\psi_{n}+l w_{n}\right), i \lambda_{n} \tilde{w}_{n}\right\rangle_{L^{2}(0,1)}= & -\left\langle i \lambda_{n} \varphi_{n x}, \tilde{w}_{n}\right\rangle_{L^{2}(0,1)}-\left\langle i \lambda_{n} \psi_{n}, \tilde{w}_{n}\right\rangle_{L^{2}(0,1)}-l\left\langle i \lambda_{n} w_{n}, \tilde{w}_{n}\right\rangle_{L^{2}(0,1)} \\
= & \left\langle\left(i \lambda_{n} \varphi_{n}-\tilde{\varphi}_{n}\right), \tilde{w}_{n x}\right\rangle_{L^{2}(0,1)}+\left\langle\tilde{\varphi}_{n}, \tilde{w}_{n x}\right\rangle_{L^{2}(0,1)}-\left\langle\left(i \lambda_{n} \psi_{n}-\tilde{\psi}_{n}\right), \tilde{w}_{n}\right\rangle_{L^{2}(0,1)} \\
& -\left\langle\tilde{\psi}_{n}, \tilde{w}_{n}\right\rangle_{L^{2}(0,1)}-l\left\langle\left(i \lambda_{n} w_{n}-\tilde{w}_{n}\right), \tilde{w}_{n}\right\rangle_{L^{2}(0,1)}-l\left\|\tilde{w}_{n}\right\|_{L^{2}(0,1)}^{2} \\
= & -\left\langle\left(i \lambda_{n} \varphi_{n x}-\tilde{\varphi}_{n x}\right), \tilde{w}_{n}\right\rangle_{L^{2}(0,1)}+\left\langle\tilde{\varphi}_{n}, \tilde{w}_{n x}\right\rangle_{L^{2}(0,1)}-\left\langle\left(i \lambda_{n} \psi_{n}-\tilde{\psi}_{n}\right), \tilde{w}_{n}\right\rangle_{L^{2}(0,1)} \\
& -\left\langle\tilde{\psi}_{n}, \tilde{w}_{n}\right\rangle_{L^{2}(0,1)}-l\left\langle\left(i \lambda_{n} w_{n}-\tilde{w}_{n}\right), \tilde{w}_{n}\right\rangle_{L^{2}(0,1)}-l\left\|\tilde{w}_{n}\right\|_{L^{2}(0,1)}^{2} .
\end{aligned}
$$


Then, using (46), (49) $1,(49)_{3},(49)_{5}$ and (59), we deduce that

$$
\left\langle\left(\varphi_{n x}+\psi_{n}+l w_{n}\right), i \lambda_{n} \tilde{w}_{n}\right\rangle_{L^{2}(0,1)}-\left\langle\tilde{\varphi}_{n}, \tilde{w}_{n x}\right\rangle_{L^{2}(0,1)} \longrightarrow 0 .
$$

Second, taking the inner product of $\tilde{\varphi}_{n}$ with $\tilde{w}_{n x}$ in $L^{2}(0,1)$, we arrive at

$$
\begin{aligned}
\left\langle\tilde{\varphi}_{n}, \tilde{w}_{n x}\right\rangle_{L^{2}(0,1)}= & \left\langle\tilde{\varphi}_{n},\left(\tilde{w}_{n x}-l \tilde{\varphi}_{n}\right)\right\rangle_{L^{2}(0,1)}+l\left\|\tilde{\varphi}_{n}\right\|_{L^{2}(0,1)}^{2} \\
= & -\left\langle\tilde{\varphi}_{n},\left(i \lambda_{n} w_{n x}-\tilde{w}_{n x}\right)\right\rangle_{L^{2}(0,1)}+\left\langle\tilde{\varphi}_{n}, l\left(i \lambda_{n} \varphi_{n}-\tilde{\varphi}_{n}\right)\right\rangle_{L^{2}(0,1)} \\
& +\left\langle\tilde{\varphi}_{n}, i \lambda_{n}\left(w_{n x}-l \varphi_{n}\right)\right\rangle_{L^{2}(0,1)}+l\left\|\tilde{\varphi}_{n}\right\|_{L^{2}(0,1)}^{2},
\end{aligned}
$$

then, by (46), (49) 1 and (49)5, we have

$$
\lambda_{n}\left\langle\tilde{\varphi}_{n}, i\left(w_{n x}-l \varphi_{n}\right)\right\rangle_{L^{2}(0,1)}-\left\langle\tilde{\varphi}_{n}, \tilde{w}_{n x}\right\rangle_{L^{2}(0,1)}+l\left\|\tilde{\varphi}_{n}\right\|_{L^{2}(0,1)}^{2} \longrightarrow 0 .
$$

Third, taking the inner product of $(49)_{2}$ with $\left(w_{n x}-l \varphi_{n}\right)$ in $L^{2}(0,1)$, integrating by parts and using the boundary conditions, we find

$$
\begin{aligned}
& \left\langle i \lambda_{n} \rho_{1} \tilde{\varphi}_{n},\left(w_{n x}-l \varphi_{n}\right)\right\rangle_{L^{2}(0,1)}+k\left\langle\left(\varphi_{n x}+\psi_{n}+l w_{n}\right),\left(w_{n x}-l \varphi_{n}\right)_{x}\right\rangle_{L^{2}(0,1)} \\
& \quad-l k_{0}\left\|\left(w_{n x}-l \varphi_{n}\right)\right\|_{L^{2}(0,1)}^{2} \longrightarrow 0
\end{aligned}
$$

which implies that

$$
\begin{aligned}
& \lambda_{n} \rho_{1}\left\langle i \tilde{\varphi}_{n},\left(w_{n x}-l \varphi_{n}\right)\right\rangle_{L^{2}(0,1)} \\
& \quad-\frac{k}{k_{0}}\left\langle\left(\varphi_{n x}+\psi_{n}+l w_{n}\right),\left[i \lambda_{n} \rho_{1} \tilde{w}_{n}-k_{0}\left(w_{n x}-l \varphi_{n}\right)_{x}+l k\left(\varphi_{n x}+\psi_{n}+l w_{n}\right)+\delta \theta_{n x}\right]\right\rangle_{L^{2}(0,1)} \\
& \quad+\frac{k \rho_{1}}{k_{0}}\left\langle\left(\varphi_{n x}+\psi_{n}+l w_{n}\right), i \lambda_{n} \tilde{w}_{n}\right\rangle_{L^{2}(0,1)}+\frac{l k^{2}}{k_{0}}\left\|\left(\varphi_{n x}+\psi_{n}+l w_{n}\right)\right\|_{L^{2}(0,1)}^{2} \\
& \quad+\frac{\delta k}{k_{0}}\left\langle\left(\varphi_{n x}+\psi_{n}+l w_{n}\right), \theta_{n x}\right\rangle_{L^{2}(0,1)}-l k_{0}\left\|\left(w_{n x}-l \varphi_{n}\right)\right\|_{L^{2}(0,1)}^{2} \longrightarrow 0 .
\end{aligned}
$$

Using (46), (49) 6 , (52), (54) and (56), we see that

$$
\begin{aligned}
& -\lambda_{n} \rho_{1}\left\langle\tilde{\varphi}_{n}, i\left(w_{n x}-l \varphi_{n}\right)\right\rangle_{L^{2}(0,1)}+\frac{k \rho_{1}}{k_{0}}\left\langle\left(\varphi_{n x}+\psi_{n}+l w_{n}\right), i \lambda_{n} \widetilde{w}_{n}\right\rangle_{L^{2}(0,1)} \\
& +\frac{l k^{2}}{k_{0}}\left\|\left(\varphi_{n x}+\psi_{n}+l w_{n}\right)\right\|_{L^{2}(0,1)}^{2} \longrightarrow 0 .
\end{aligned}
$$

Then, multiplying (61) by $\frac{-k \rho_{1}}{k_{0}}$ and (62) by $\rho_{1}$, and adding the obtained limits and (63), we obtain

$$
\left(\frac{k}{k_{0}}-1\right) \rho_{1}\left\langle\tilde{\varphi}_{n}, \tilde{w}_{n x}\right\rangle_{L^{2}(0,1)}+\frac{l k^{2}}{k_{0}}\left\|\left(\varphi_{n x}+\psi_{n}+l w_{n}\right)\right\|_{L^{2}(0,1)}^{2}+\rho_{1} l\left\|\tilde{\varphi}_{n}\right\|_{L^{2}(0,1)}^{2} \longrightarrow 0 .
$$

So, because $k=k_{0}$ (according to (25)), we get from (54) and (64) that

$$
\varphi_{n x} \longrightarrow 0 \text { in } L^{2}(0,1)
$$

and

$$
\tilde{\varphi}_{n} \longrightarrow 0 \text { in } L^{2}(0,1) \text {. }
$$

Moreover, (49) 1 and (66) give

$$
\lambda_{n} \varphi_{n} \longrightarrow 0 \text { in } L^{2}(0,1) .
$$


Estimates on $\tilde{\psi}_{n}$ and $\lambda_{n} \psi_{n}$ First, taking the inner product of $(49)_{4}$ with $\left(\varphi_{n x}+\psi_{n}+l w_{n}\right)$ in $L^{2}(0,1)$, integrating by parts and using the boundary conditions, we get

$$
\begin{aligned}
& \left\langle i \lambda_{n} \rho_{2} \tilde{\psi}_{n}, \varphi_{n x}\right\rangle_{L^{2}(0,1)}+\left\langle i \lambda_{n} \rho_{2} \tilde{\psi}_{n}, \psi_{n}\right\rangle_{L^{2}(0,1)}+l\left\langle i \lambda_{n} \rho_{2} \tilde{\psi}_{n}, w_{n}\right\rangle_{L^{2}(0,1)} \\
& \quad+b\left\langle\psi_{n x},\left(\varphi_{n x}+\psi_{n}+l w_{n}\right)_{x}\right\rangle_{L^{2}(0,1)}+k\left\|\left(\varphi_{n x}+\psi_{n}+l w_{n}\right)\right\|_{L^{2}(0,1)}^{2} \longrightarrow 0,
\end{aligned}
$$

then

$$
\begin{aligned}
- & \lambda_{n} \rho_{2}\left\langle\tilde{\psi}_{n}, i \varphi_{n x}\right\rangle_{L^{2}(0,1)}-\rho_{2}\left\langle\tilde{\psi}_{n},\left(i \lambda_{n} \psi_{n}-\tilde{\psi}_{n}\right)\right\rangle_{L^{2}(0,1)}-\rho_{2}\left\|\tilde{\psi}_{n}\right\|_{L^{2}(0,1)}^{2} \\
& -l \rho_{2}\left\langle\tilde{\psi}_{n},\left(i \lambda_{n} w_{n}-\tilde{w}_{n}\right)\right\rangle_{L^{2}(0,1)}-l \rho_{2}\left\langle\tilde{\psi}_{n}, \tilde{w}_{n}\right\rangle_{L^{2}(0,1)} \\
& -\frac{b}{k}\left\langle\psi_{n x},\left[i \lambda_{n} \rho_{1} \tilde{\varphi}_{n}-k\left(\varphi_{n x}+\psi_{n}+l w_{n}\right)_{x}-l k_{0}\left(w_{n x}-l \varphi_{n}\right)\right]\right\rangle_{L^{2}(0,1)} \\
& +\frac{b}{k}\left\langle\psi_{n x}, i \lambda_{n} \rho_{1} \tilde{\varphi}_{n}\right\rangle_{L^{2}(0,1)}-\frac{l k_{0} b}{k}\left\langle\psi_{n x},\left(w_{n x}-l \varphi_{n}\right)\right\rangle_{L^{2}(0,1)}+k\left\|\varphi_{n x}+\psi_{n}+l w_{n}\right\|_{L^{2}(0,1)}^{2} \longrightarrow 0,
\end{aligned}
$$

using (46), (49) $2,(49)_{3},(49)_{5},(54),(56),(59)$ and (65), we get

$$
-\lambda_{n} \rho_{2}\left\langle\tilde{\psi}_{n}, i \varphi_{n x}\right\rangle_{L^{2}(0,1)}-\rho_{2}\left\|\tilde{\psi}_{n}\right\|_{L^{2}(0,1)}^{2}+\frac{b \rho_{1}}{k} \lambda_{n}\left\langle\psi_{n x}, i \tilde{\varphi}_{n}\right\rangle_{L^{2}(0,1)} \longrightarrow 0 .
$$

Second, using the equality

$$
\lambda_{n}\left\langle\psi_{n x}, i \tilde{\varphi}_{n}\right\rangle_{L^{2}(0,1)}=-\left\langle\left(i \lambda_{n} \psi_{n x}-\tilde{\psi}_{n x}\right), \tilde{\varphi}_{n}\right\rangle_{L^{2}(0,1)}-\left\langle\tilde{\psi}_{n x}, \tilde{\varphi}_{n}\right\rangle_{L^{2}(0,1)},
$$

integrating by parts and using the boundary conditions, we obtain

$$
\begin{aligned}
\lambda_{n}\left\langle\psi_{n x}, i \tilde{\varphi}_{n}\right\rangle_{L^{2}(0,1)} & =-\left\langle\left(i \lambda_{n} \psi_{n x}-\tilde{\psi}_{n x}\right), \tilde{\varphi}_{n}\right\rangle_{L^{2}(0,1)}+\left\langle\tilde{\psi}_{n}, \tilde{\varphi}_{n x}\right\rangle_{L^{2}(0,1)} \\
& =-\left\langle\left(i \lambda_{n} \psi_{n x}-\tilde{\psi}_{n x}\right), \tilde{\varphi}_{n}\right\rangle_{L^{2}(0,1)}-\left\langle\tilde{\psi}_{n},\left(i \lambda_{n} \varphi_{n x}-\tilde{\varphi}_{n x}\right)\right\rangle_{L^{2}(0,1)}+\left\langle\tilde{\psi}_{n}, i \lambda_{n} \varphi_{n x}\right\rangle_{L^{2}(0,1)} .
\end{aligned}
$$

Therefore, from (46), (49) 1 and (49) $)_{3}$, we see that

$$
\lambda_{n}\left\langle\psi_{n x}, i \tilde{\varphi}_{n}\right\rangle_{L^{2}(0,1)}-\lambda_{n}\left\langle\tilde{\psi}_{n}, i \varphi_{n x}\right\rangle_{L^{2}(0,1)} \longrightarrow 0
$$

so, multiplying (69) by $-\rho_{2}$ and inserting the obtained limit into (68), we obtain

$$
\frac{\lambda_{n}}{k}\left(b \rho_{1}-k \rho_{2}\right)\left\langle\psi_{n x}, i \tilde{\varphi}_{n}\right\rangle_{L^{2}(0,1)}-\rho_{2}\left\|\tilde{\psi}_{n}\right\|_{L^{2}(0,1)}^{2} \longrightarrow 0 .
$$

Now, we use the fact that $b \rho_{1}-k \rho_{2}=0$ (Condition (25)), we get from (70) that

$$
\tilde{\psi}_{n} \longrightarrow 0 \text { in } L^{2}(0,1)
$$

and by $(49)_{3}$ and (71), we deduce that

$$
\lambda_{n} \psi_{n} \longrightarrow 0 \text { in } L^{2}(0,1) .
$$


Estimate on $\psi_{n x}$ and conclusion Taking the inner product of (49) 4 with $\psi_{n}$ in $L^{2}(0,1)$, integrating by parts and using the boundary conditions, we get

$$
-\rho_{2}\left\langle\tilde{\psi}_{n}, i \lambda_{n} \psi_{n}\right\rangle_{L^{2}(0,1)}+b\left\|\psi_{n x}\right\|_{L^{2}(0,1)}^{2}+k\left\langle\left(\varphi_{n x}+\psi_{n}+l w_{n}\right), \psi_{n}\right\rangle_{L^{2}(0,1)} \longrightarrow 0,
$$

and using (46), (54) and (72), we obtain

$$
\psi_{n x} \longrightarrow 0 \text { in } L^{2}(0,1)
$$

A combination of (53), (54), (56), (59), (65), (66), (71) and (73) leads to (50), which is a contradiction with (46). Hence, in case (1), (25) implies (27).

\subsubsection{Case of System (4)}

In case (4), the limit (48) implies the following ones:

$$
\left\{\begin{array}{l}
i \lambda_{n} \varphi_{n}-\tilde{\varphi}_{n} \longrightarrow 0 \text { in } H_{*}^{1}(0,1) \\
i \lambda_{n} \rho_{1} \tilde{\varphi}_{n}-k\left(\varphi_{n x}+\psi_{n}+l w_{n}\right)_{x}-l k_{0}\left(w_{n x}-l \varphi_{n}\right) \longrightarrow 0 \text { in } L^{2}(0,1), \\
i \lambda_{n} \psi_{n}-\tilde{\psi}_{n} \longrightarrow 0 \text { in } \tilde{H}_{*}^{1}(0,1), \\
i \lambda_{n} \rho_{2} \tilde{\psi}_{n}-b \psi_{n x x}+k\left(\varphi_{n x}+\psi_{n}+l w_{n}\right) \longrightarrow 0 \text { in } L^{2}(0,1), \\
i \lambda_{n} w_{n}-\tilde{w}_{n} \longrightarrow 0 \text { in } \tilde{H}_{*}^{1}(0,1), \\
i \lambda_{n} \rho_{1} \tilde{w}_{n}-k_{0}\left(w_{n x}-l \varphi_{n}\right)_{x}+l k\left(\varphi_{n x}+\psi_{n}+l w_{n}\right)+\delta \tilde{\theta}_{n x} \longrightarrow 0 \text { in } L^{2}(0,1), \\
i \lambda_{n} \theta_{n}-\tilde{\theta}_{n} \longrightarrow 0 \text { in } H_{*}^{1}(0,1), \\
i \lambda_{n} \rho_{3} \tilde{\theta}_{n}-\left(\beta \theta_{n}+\gamma \tilde{\theta}_{n}\right)_{x x}+\delta \tilde{w}_{n x} \longrightarrow 0 \text { in } L^{2}(0,1) .
\end{array}\right.
$$

Estimates on $\lambda_{n} \theta_{n}, \lambda_{n} \theta_{n x}, \tilde{\theta}_{n}$ and $\tilde{\theta}_{n x}$ Taking the inner product of $\left(i \lambda_{n} I-\mathcal{A}\right) \Phi_{n}$ with $\Phi_{n}$ in $\mathcal{H}$ and using (17), we find

$$
\operatorname{Re}\left\langle\left(i \lambda_{n} I-\mathcal{A}\right) \Phi_{n}, \Phi_{n}\right\rangle_{\mathcal{H}}=\gamma\left\|\tilde{\theta}_{n x}\right\|_{L^{2}(0,1)}^{2} .
$$

Using (46) and (48), we deduce that

$$
\tilde{\theta}_{n x} \longrightarrow 0 \text { in } L^{2}(0,1)
$$

Because $\tilde{\theta}_{n}(0)=0$ and according to Poincaré's inequality, then we get from (76) that

$$
\tilde{\theta}_{n} \longrightarrow 0 \text { in } L^{2}(0,1) \text {. }
$$

The above two limits combined with $(74)_{7}$ give

$$
\lambda_{n} \theta_{n x} \longrightarrow 0 \text { in } L^{2}(0,1)
$$

and

$$
\lambda_{n} \theta_{n} \longrightarrow 0 \text { in } L^{2}(0,1) .
$$

Estimates on $\varphi_{n}, \psi_{n}$ and $w_{n}$ Multiplying $(74)_{1},(74)_{3}$ and (74) 5 by $\frac{1}{\lambda_{n}}$, and using (46) and (47), we find (54).

Estimate on $\frac{1}{\lambda_{n}} w_{n x x}$ As in case (1) (Sect. 3.2.1), applying triangle inequality and using (74)6 and (76), we obtain (55). 
Estimates on $w_{n x}, \frac{1}{\lambda_{n}} \widetilde{w}_{n x}$ and $\frac{1}{\lambda_{n}} \widetilde{w}_{n}$ Taking the inner product of $(74)_{8}$ with $\frac{i w_{n x}}{\lambda_{n}}$ in $L^{2}(0,1)$, integrating by parts and using (46), (47) and the boundary conditions, we get

$$
\begin{aligned}
& \rho_{3}\left\langle\tilde{\theta}_{n}, w_{n x}\right\rangle_{L^{2}(0,1)}+\beta\left\langle\theta_{n x}, \frac{i w_{n x x}}{\lambda_{n}}\right\rangle_{L^{2}(0,1)}+\gamma\left\langle\tilde{\theta}_{n x}, \frac{i w_{n x x}}{\lambda_{n}}\right\rangle_{L^{2}(0,1)} \\
& -\delta\left\langle\left(i \lambda_{n} w_{n x}-\tilde{w}_{n x}\right), \frac{i w_{n x}}{\lambda_{n}}\right\rangle_{L^{2}(0,1)}+\delta\left\|w_{n x}\right\|_{L^{2}(0,1)}^{2} \longrightarrow 0
\end{aligned}
$$

Using (55), (74)5, (76), (77) and (78), we get (56). By multiplying (74) 5 by $\frac{1}{\lambda_{n}}$, we get (57). Moreover, because $\widetilde{w}_{n}(1)=0$, we have $(58)$.

Estimates on $\tilde{w}_{n}$ and $\lambda_{n} w_{n}$ As in case (1) (Sect. 3.2.1), taking the inner product of (74) 6 with $\frac{i \tilde{w}_{n}}{\lambda_{n}}$ in $L^{2}(0,1)$, integrating by parts and using the boundary conditions, we find (59) and (60).

Estimate on $\tilde{\varphi}_{n}$ and conclusion The same computations as in case (1) (Sect. 3.2.1) imply (64) and (70), so (25) leads to (65), (66), (71) and (73). Consequently, (50) holds, which is a contradiction with (46). Hence, also in case (4), (25) implies (27).

\subsection{Condition (27) implies (25)}

We prove this implication by contradiction. So, we assume that (25) does not hold and prove that (27) is not satisfied; that is we prove that there exists a sequence $\left(\lambda_{n}\right)_{n} \subset \mathbb{R}$ such that

$$
\lim _{n \rightarrow \infty}\left\|\left(i \lambda_{n} I-\mathcal{A}\right)^{-1}\right\|_{\mathcal{L}(\mathcal{H})}=\infty
$$

which is equivalent to prove that there exists a sequence $\left(F_{n}\right)_{n} \subset \mathcal{H}$ satisfying

$$
\left\|F_{n}\right\|_{\mathcal{H}} \leq 1, \quad \forall n \in \mathbb{N}
$$

and

$$
\lim _{n \rightarrow \infty}\left\|\left(i \lambda_{n} I-\mathcal{A}\right)^{-1} F_{n}\right\|_{\mathcal{H}}=\infty
$$

For this purpose, let

$$
\Phi_{n}=\left(i \lambda_{n} I-\mathcal{A}\right)^{-1} F_{n}, \quad \forall n \in \mathbb{N} .
$$

Then we have to prove that (80) holds such that

$$
\lim _{n \rightarrow \infty}\left\|\Phi_{n}\right\|_{\mathcal{H}}=\infty \text { and } i \lambda_{n} \Phi_{n}-\mathcal{A} \Phi_{n}=F_{n}, \quad \forall n \in \mathbb{N}
$$

Taking

$$
\Phi_{n}= \begin{cases}\left(\varphi_{n}, \tilde{\varphi}_{n}, \psi_{n}, \tilde{\psi}_{n}, w_{n}, \tilde{w}_{n}, \theta_{n}\right)^{T} & \text { in case (1) } \\ \left(\varphi_{n}, \tilde{\varphi}_{n}, \psi_{n}, \tilde{\psi}_{n}, w_{n}, \tilde{w}_{n}, \theta_{n}, \tilde{\theta}_{n}\right)^{T} & \text { in case (4) }\end{cases}
$$

and

$$
F_{n}= \begin{cases}\left(f_{1 n}, \ldots, f_{7 n}\right)^{T} & \text { in case (1) } \\ \left(f_{1 n}, \ldots, f_{8 n}\right)^{T} & \text { in case (4) }\end{cases}
$$


Then, from the second equality in (82), we have the following systems:

$$
\left\{\begin{array}{l}
i \lambda_{n} \varphi_{n}-\tilde{\varphi}_{n}=f_{1 n}, \\
i \rho_{1} \lambda_{n} \tilde{\varphi}_{n}-k\left(\varphi_{n x}+\psi_{n}+l w_{n}\right)_{x}-l k_{0}\left(w_{n x}-l \varphi_{n}\right)=\rho_{1} f_{2 n}, \\
i \lambda_{n} \psi_{n}-\tilde{\psi}_{n}=f_{3 n}, \\
i \rho_{2} \lambda_{n} \tilde{\psi}_{n}-b \psi_{n x x}+k\left(\varphi_{n x}+\psi_{n}+l w_{n}\right)=\rho_{2} f_{4 n}, \\
i \lambda_{n} w_{n}-\tilde{w}_{n}=f_{5 n}, \\
i \rho_{1} \lambda_{n} \tilde{w}_{n}-k_{0}\left(w_{n x}-l \varphi_{n}\right)_{x}+l k\left(\varphi_{n x}+\psi_{n}+l w_{n}\right)+\delta \theta_{n x}=\rho_{1} f_{6 n}, \\
i \rho_{3} \lambda_{n} \theta_{n}-\beta \theta_{n x x}+\delta \tilde{w}_{n x}=\rho_{3} f_{7 n}
\end{array}\right.
$$

in case (1), and

$$
\left\{\begin{array}{l}
i \lambda_{n} \varphi_{n}-\tilde{\varphi}_{n}=f_{1 n}, \\
i \rho_{1} \lambda_{n} \tilde{\varphi}_{n}-k\left(\varphi_{n x}+\psi_{n}+l w_{n}\right)_{x}-l k_{0}\left(w_{n x}-l \varphi_{n}\right)=\rho_{1} f_{2 n}, \\
i \lambda_{n} \psi_{n}-\tilde{\psi}_{n}=f_{3 n}, \\
i \rho_{2} \lambda_{n} \tilde{\psi}_{n}-b \psi_{n x x}+k\left(\varphi_{n x}+\psi_{n}+l w_{n}\right)=\rho_{2} f_{4 n}, \\
i \lambda_{n} w_{n}-\tilde{w}_{n}=f_{5 n}, \\
i \rho_{1} \lambda_{n} \tilde{w}_{n}-k_{0}\left(w_{n x}-l \varphi_{n}\right)_{x}+l k\left(\varphi_{n x}+\psi_{n}+l w_{n}\right)+\delta \tilde{\theta}_{n x}=\rho_{1} f_{6 n}, \\
i \lambda_{n} \theta_{n}-\tilde{\theta}_{n}=f_{7 n}, \\
i \rho_{3} \lambda_{n} \tilde{\theta}_{n}-\left(\beta \theta_{n}+\gamma \tilde{\theta}_{n}\right)_{x x}+\delta \tilde{w}_{n x}=\rho_{3} f_{8 n}
\end{array}\right.
$$

in case (4). Choosing

$$
f_{4 n}(x)=c \cos (N x), \quad f_{1 n}=f_{2 n}=f_{3 n}=f_{5 n}=f_{6 n}(x)=f_{7 n}=f_{8 n}=0,
$$

where $N=\frac{(2 n+1) \pi}{2}$ and $c$ is a constant satisfying $0<|c| \leq \frac{1}{\sqrt{\rho_{2}}}$, so

$$
\left\|F_{n}\right\|_{\mathcal{H}}^{2}=\rho_{2}\left\|f_{4 n}\right\|_{L^{2}(0,1)}^{2}=\rho_{2}|c|^{2} \int_{0}^{1} \cos ^{2}(N x) \mathrm{d} x \leq 1 .
$$

On the other hand, the systems (83) and (84) become, respectively,

$$
\left\{\begin{array}{l}
\tilde{\varphi}_{n}=i \lambda_{n} \varphi_{n}, \quad \tilde{\psi}_{n}=i \lambda_{n} \psi_{n}, \quad \tilde{w}_{n}=i \lambda_{n} w_{n}, \\
-\rho_{1} \lambda_{n}^{2} \varphi_{n}-k\left(\varphi_{n x}+\psi_{n}+l w_{n}\right)_{x}-l k_{0}\left(w_{n x}-l \varphi_{n}\right)=0, \\
-\rho_{2} \lambda_{n}^{2} \psi_{n}-b \psi_{n x x}+k\left(\varphi_{n x}+\psi_{n}+l w_{n}\right)=\rho_{2} f_{4 n}, \\
-\rho_{1} \lambda_{n}^{2} w_{n}-k_{0}\left(w_{n x}-l \varphi_{n}\right)_{x}+l k\left(\varphi_{n x}+\psi_{n}+l w_{n}\right)+\delta \theta_{n x}=0, \\
i \rho_{3} \lambda_{n} \theta_{n}-\beta \theta_{n x x}+i \delta \lambda_{n} w_{n x}=0
\end{array}\right.
$$

and

$$
\left\{\begin{array}{l}
\tilde{\varphi}_{n}=i \lambda_{n} \varphi_{n}, \quad \tilde{\psi}_{n}=i \lambda_{n} \psi_{n}, \quad \tilde{w}_{n}=i \lambda_{n} w_{n}, \quad \tilde{\theta}_{n}=i \lambda_{n} \theta_{n}, \\
-\rho_{1} \lambda_{n}^{2} \varphi_{n}-k\left(\varphi_{n x}+\psi_{n}+l w_{n}\right)_{x}-l k_{0}\left(w_{n x}-l \varphi_{n}\right)=0, \\
-\rho_{2} \lambda_{n}^{2} \psi_{n}-b \psi_{n x x}+k\left(\varphi_{n x}+\psi_{n}+l w_{n}\right)=\rho_{2} f_{4 n}, \\
-\rho_{1} \lambda_{n}^{2} w_{n}-k_{0}\left(w_{n x}-l \varphi_{n}\right)_{x}+l k\left(\varphi_{n x}+\psi_{n}+l w_{n}\right)+i \delta \lambda_{n} \theta_{n x}=0, \\
-i \rho_{3} \lambda_{n}^{2} \theta_{n}-\left(\beta \theta_{n}+i \gamma \lambda_{n} \theta_{n}\right)_{x x}+i \delta \lambda_{n} w_{n x}=0 .
\end{array}\right.
$$


Let us consider the choices

$$
\left\{\begin{array}{l}
\varphi_{n}(x)=\alpha_{1} \sin (N x), \psi_{n}(x)=\alpha_{2} \cos (N x), w_{n}(x)=\alpha_{3} \cos (N x), \\
\theta_{n}(x)=\alpha_{4} \sin (N x), \tilde{\varphi}_{n}(x)=i \lambda_{n} \alpha_{1} \sin (N x), \tilde{\psi}_{n}(x)=i \lambda_{n} \alpha_{2} \cos (N x), \\
\tilde{w}_{n}(x)=i \lambda_{n} \alpha_{3} \cos (N x), \tilde{\theta}_{n}(x)=i \lambda_{n} \alpha_{4} \sin (N x),
\end{array}\right.
$$

where $\alpha_{1}, \ldots, \alpha_{4}$ are constants depending on $N$ (will be fixed later). Then the last equation in (86) and the last one in (87) are equivalent to $\alpha_{4}=\mu_{n} N \alpha_{3}$, where

$$
\mu_{n}= \begin{cases}\frac{i \delta \lambda_{n}}{\beta N^{2}+i \rho_{3} \lambda_{n}} & \text { in case (86), } \\ \frac{i \delta \lambda_{n}}{i \gamma \lambda_{n} N^{2}+\beta N^{2}-i \rho_{3} \lambda_{n}^{2}} & \text { in case (87). }\end{cases}
$$

Therefore, (86) and (87) are satisfied if and only if

$$
\left\{\begin{array}{l}
{\left[k N^{2}+l^{2} k_{0}-\rho_{1} \lambda_{n}^{2}\right] \alpha_{1}+k N \alpha_{2}+l\left(k+k_{0}\right) N \alpha_{3}=0,} \\
{\left[b N^{2}+k-\rho_{2} \lambda_{n}^{2}\right] \alpha_{2}+k N \alpha_{1}+l k \alpha_{3}=\rho_{2} c,} \\
{\left[\left(k_{0}+\delta_{n} \mu_{n}\right) N^{2}+l^{2} k-\rho_{1} \lambda_{n}^{2}\right] \alpha_{3}+l\left(k+k_{0}\right) N \alpha_{1}+l k \alpha_{2}=0,}
\end{array}\right.
$$

where

$$
\delta_{n}= \begin{cases}\delta & \text { in case (86) } \\ i \delta \lambda_{n} & \text { in case (87) }\end{cases}
$$

Because (25) is assumed to be not satisfied, then

$$
\rho_{1} b-\rho_{2} k \neq 0 \text { or }\left[\rho_{1} b-\rho_{2} k=0 \text { and } k-k_{0} \neq 0\right],
$$

so we distinguish these two cases.

Case $1 \rho_{1} b-\rho_{2} k \neq 0$. Let choose $\lambda_{n}=\sqrt{\frac{b}{\rho_{2}} N^{2}+\frac{k k_{0}}{\rho_{2}\left(k+k_{0}\right)}}$, then

$$
\lim _{n \rightarrow \infty} \delta_{n} \mu_{n}=0 \quad \text { and } \quad N^{2} \delta_{n} \mu_{n} \sim \begin{cases}\frac{i \delta^{2}}{\beta} \lambda_{n} & \text { in case (86), } \\ \frac{i \delta^{2}}{\gamma} \lambda_{n} & \text { in case (87). }\end{cases}
$$

On the other hand, (89) becomes

$$
\left\{\begin{array}{l}
{\left[\left(k-\frac{\rho_{1} b}{\rho_{2}}\right) N^{2}+l^{2} k_{0}-\frac{\rho_{1} k k_{0}}{\rho_{2}\left(k+k_{0}\right)}\right] \alpha_{1}+k N \alpha_{2}+l\left(k+k_{0}\right) N \alpha_{3}=0,} \\
\frac{k^{2}}{k+k_{0}} \alpha_{2}+k N \alpha_{1}+l k \alpha_{3}=\rho_{2} c, \\
{\left[\left(k_{0}-\frac{\rho_{1} b}{\rho_{2}}+\delta_{n} \mu_{n}\right) N^{2}+l^{2} k-\frac{\rho_{1} k k_{0}}{\rho_{2}\left(k+k_{0}\right)}\right] \alpha_{3}+l\left(k+k_{0}\right) N \alpha_{1}+l k \alpha_{2}=0 .}
\end{array}\right.
$$

From $(91)_{2}$ we get

$$
\alpha_{1}=\frac{\rho_{2} c-l k \alpha_{3}-\frac{k^{2}}{k+k_{0}} \alpha_{2}}{k N} .
$$

By substituting (92) into $(91)_{3}$ and into $(91)_{1}$, we obtain, respectively,

$$
\alpha_{3}=\frac{\rho_{2} l c\left(k+k_{0}\right)}{k\left[\left(\frac{\rho_{1} b}{\rho_{2}}-k_{0}-\delta_{n} \mu_{n}\right) N^{2}+l^{2} k_{0}+\frac{\rho_{1} k k_{0}}{\rho_{2}\left(k+k_{0}\right)}\right]}
$$


and

$$
\alpha_{2}=\frac{\left[\left(\rho_{2} c-l k \alpha_{3}\right)\left(k-\frac{\rho_{1} b}{\rho_{2}}\right)+l k\left(k+k_{0}\right) \alpha_{3}\right] N^{2}+\left(\rho_{2} c-l k \alpha_{3}\right)\left[l^{2} k_{0}-\frac{\rho_{1} k k_{0}}{\rho_{2}\left(k+k_{0}\right)}\right]}{\frac{k^{2}}{k+k_{0}}\left[-\left(\frac{\rho_{1} b}{\rho_{2}}+k_{0}\right) N^{2}+l^{2} k_{0}-\frac{\rho_{1} k k_{0}}{\rho_{2}\left(k+k_{0}\right)}\right]} .
$$

According to (90), we see that (93) implies that

$$
\lim _{n \rightarrow \infty} \alpha_{3}=0
$$

therefore,

$$
\lim _{n \rightarrow \infty} \alpha_{2}=\frac{c\left(k+k_{0}\right)\left(\rho_{1} b-\rho_{2} k\right)}{k^{2}\left(\frac{\rho_{1} b}{\rho_{2}}+k_{0}\right)} \neq 0
$$

since $\rho_{1} b-\rho_{2} k \neq 0$. Then

$$
\lim _{n \rightarrow \infty}\left|\alpha_{2}\right| N=\infty
$$

Finally, using the norm of $\psi_{n x}$ in $L^{2}(0,1)$, we obtain

$$
\begin{aligned}
\left\|\Phi_{n}\right\|_{\mathcal{H}}^{2} \geq b\left\|\psi_{n x}\right\|_{L^{2}(0,1)}^{2} & =b\left|\alpha_{2}\right|^{2} N^{2} \int_{0}^{1} \sin ^{2}(N x) \mathrm{d} x \\
& \geq \frac{b}{2}\left|\alpha_{2}\right|^{2} N^{2} \int_{0}^{1}(1-\cos (2 N x)) \mathrm{d} x=\frac{b}{2}\left|\alpha_{2}\right|^{2} N^{2} \longrightarrow \infty
\end{aligned}
$$

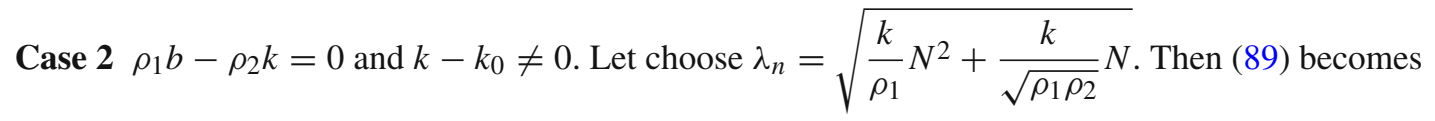

$$
\left\{\begin{array}{l}
\left(-\frac{\rho_{1} k}{\sqrt{\rho_{1} \rho_{2}}} N+l^{2} k_{0}\right) \alpha_{1}+k N \alpha_{2}+l\left(k+k_{0}\right) N \alpha_{3}=0, \\
\left(-\frac{\rho_{2} k}{\sqrt{\rho_{1} \rho_{2}}} N+k\right) \alpha_{2}+k N \alpha_{1}+l k \alpha_{3}=\rho_{2} c, \\
{\left[\left(k_{0}-k+\delta_{n} \mu_{n}\right) N^{2}-\frac{\rho_{1} k}{\sqrt{\rho_{1} \rho_{2}}} N+l^{2} k\right] \alpha_{3}+l\left(k+k_{0}\right) N \alpha_{1}+l k \alpha_{2}=0 .}
\end{array}\right.
$$

From $(97)_{1}$ we get, for $N>\frac{l^{2} k_{0} \sqrt{\rho_{1} \rho_{2}}}{\rho_{1} k}$,

$$
\alpha_{1}=\frac{k N \alpha_{2}+l\left(k+k_{0}\right) N \alpha_{3}}{\frac{\rho_{1} k}{\sqrt{\rho_{1} \rho_{2}}} N-l^{2} k_{0}} .
$$

By substituting (98) into $(97)_{3}$, we find, for $N>\frac{l^{2} k_{0} \sqrt{\rho_{1} \rho_{2}}}{\rho_{1} k}$,

$$
\alpha_{3}=\frac{l k\left[\left(k+k_{0}\right) N^{2}+\frac{\rho_{1} k}{\sqrt{\rho_{1} \rho_{2}}} N-l^{2} k_{0}\right] \alpha_{2}}{\left(-\frac{\rho_{1} k}{\sqrt{\rho_{1} \rho_{2}}} N+l^{2} k_{0}\right)\left[\left(k_{0}-k+\delta_{n} \mu_{n}\right) N^{2}-\frac{\rho_{1} k}{\sqrt{\rho_{1} \rho_{2}}} N+l^{2} k\right]-l^{2}\left(k+k_{0}\right)^{2} N^{2}} .
$$

By substituting (98) and (99) into (97) 2 , we obtain, for $N>\frac{l^{2} k_{0} \sqrt{\rho_{1} \rho_{2}}}{\rho_{1} k}$,

$$
\alpha_{2}=\frac{a_{1}}{a_{2}}
$$


where

$$
\begin{aligned}
a_{1}= & -\rho_{2} c\left(\frac{\rho_{1} k}{\sqrt{\rho_{1} \rho_{2}}} N-l^{2} k_{0}\right)^{2}\left[\left(k_{0}-k+\delta_{n} \mu_{n}\right) N^{2}-\frac{\rho_{1} k}{\sqrt{\rho_{1} \rho_{2}}} N+l^{2} k\right] \\
& +\rho_{2} c l^{2}\left(k+k_{0}\right)^{2}\left(-\frac{\rho_{1} k}{\sqrt{\rho_{1} \rho_{2}}} N+l^{2} k_{0}\right) N^{2}
\end{aligned}
$$

and

$$
\begin{aligned}
a_{2}= & l^{2} k^{2}\left[\left(k+k_{0}\right) N^{2}+\frac{\rho_{1} k}{\sqrt{\rho_{1} \rho_{2}}} N-l^{2} k_{0}\right]^{2}+l^{2}\left(k+k_{0}\right)^{2}\left(l^{2} k k_{0}-\frac{\rho_{1} k^{2}+l^{2} k k_{0} \rho_{2}}{\sqrt{\rho_{1} \rho_{2}}} N\right) N^{2} \\
& +\left(l^{2} k k_{0}-\frac{\rho_{1} k^{2}+l^{2} k k_{0} \rho_{2}}{\sqrt{\rho_{1} \rho_{2}}} N\right)\left(\frac{\rho_{1} k}{\sqrt{\rho_{1} \rho_{2}}} N-l^{2} k_{0}\right)\left[\left(k_{0}-k+\delta_{n} \mu_{n}\right) N^{2}-\frac{\rho_{1} k}{\sqrt{\rho_{1} \rho_{2}}} N+l^{2} k\right] .
\end{aligned}
$$

We see that (90) and (100) imply that

$$
\lim _{n \rightarrow \infty}\left|\alpha_{2}\right|= \begin{cases}\left|\frac{c \rho_{1} \rho_{2}\left(k-k_{0}\right)}{k\left[\rho_{2} l^{2}\left(k+3 k_{0}\right)+\rho_{1}\left(k-k_{0}\right)\right]}\right| & \text { if } \rho_{2} l^{2}\left(k+3 k_{0}\right)+\rho_{1}\left(k-k_{0}\right) \neq 0, \\ \infty & \text { if } \rho_{2} l^{2}\left(k+3 k_{0}\right)+\rho_{1}\left(k-k_{0}\right)=0 .\end{cases}
$$

Because $k-k_{0} \neq 0$, then (95) holds. Consequently, (96) remains valid.

Finally, the equivalence between (27) and (25) is established, and consequently, the proof of Theorem 3.1 is completed.

\section{Polynomial stability}

In this section, we prove the following polynomial stability independently from (25):

Theorem 4.1 Assume that (15) and (24) hold. Then, for any $m \in \mathbb{N}^{*}$, there exists a constant $c_{m}>0$ such that, for any $\Phi_{0} \in D\left(\mathcal{A}^{m}\right)$ and $t>0$,

$$
\left\|e^{t \mathcal{A}} \Phi_{0}\right\|_{\mathcal{H}} \leq \begin{cases}c_{m}\left\|\Phi_{0}\right\|_{D\left(\mathcal{A}^{m}\right)}\left(\frac{\ln t}{t}\right) \frac{m}{4} \ln t & \text { if } \rho_{1} b-\rho_{2} k=0, \\ c_{m}\left\|\Phi_{0}\right\|_{D\left(\mathcal{A}^{m}\right)}\left(\frac{\ln t}{t}\right) \frac{m}{10} \ln t & \text { if } \rho_{1} b-\rho_{2} k \neq 0 .\end{cases}
$$

The key of the proof of Theorem 4.1 is the following known theorem:

Theorem 4.2 [12] If a bounded $C_{0}$ semigroup $e^{t \mathcal{A}}$ on a Hilbert space $\mathcal{H}$ generated by an operator $\mathcal{A}$ satisfies (26) and, for some $j \in \mathbb{N}^{*}$,

$$
\sup _{|\lambda| \geq 1} \frac{1}{\lambda^{j}}\left\|(i \lambda I-\mathcal{A})^{-1}\right\|_{\mathcal{L}(\mathcal{H})}<\infty .
$$

Then, for any $m \in \mathbb{N}^{*}$, there exists a positive constant $c_{m}$ such that

$$
\left\|e^{t \mathcal{A}} z_{0}\right\|_{\mathcal{H}} \leq c_{m}\left\|z_{0}\right\|_{D\left(\mathcal{A}^{m}\right)}\left(\frac{\ln t}{t}\right)^{\frac{m}{j}} \ln t, \quad \forall z_{0} \in D\left(\mathcal{A}^{m}\right), \quad \forall t>0 .
$$

Proof In Sect. 3, we have proved that (24) implies (26). Then we only need to show (103), where $j=4$ if $\rho_{1} b-\rho_{2} k=0$, and $j=10$ if $\rho_{1} b-\rho_{2} k \neq 0$. Let us establish (103) by contradiction. Assume that (103) is false, then there exist sequences $\left(\Phi_{n}\right)_{n} \subset D(\mathcal{A})$ and $\left(\lambda_{n}\right)_{n} \subset \mathbb{R}$ satisfying (46), (47) and

$$
\lim _{n \rightarrow \infty} \lambda_{n}^{j}\left\|\left(i \lambda_{n} I-\mathcal{A}\right) \Phi_{n}\right\|_{\mathcal{H}}=0 .
$$

To get a contradiction with (46), we use similar arguments to the ones used in Sect. 3.2. Let

$$
\Phi_{n}= \begin{cases}\left(\varphi_{n}, \tilde{\varphi}_{n}, \psi_{n}, \tilde{\psi}_{n}, w_{n}, \tilde{w}_{n}, \theta_{n}\right)^{T} & \text { in case (1) } \\ \left(\varphi_{n}, \tilde{\varphi}_{n}, \psi_{n}, \tilde{\psi}_{n}, w_{n}, \tilde{w}_{n}, \theta_{n}, \tilde{\theta}_{n}\right)^{T} & \text { in case (4). }\end{cases}
$$


4.1 Case of System (1) with $\rho_{1} b-\rho_{2} k=0$

The limit (105) with $j=4$ implies that

$$
\left\{\begin{array}{l}
\lambda_{n}^{4}\left[i \lambda_{n} \varphi_{n}-\tilde{\varphi}_{n}\right] \rightarrow 0 \text { in } H_{*}^{1}(0,1) \\
\lambda_{n}^{4}\left[i \rho_{1} \lambda_{n} \tilde{\varphi}_{n}-k\left(\varphi_{n x}+\psi_{n}+l w_{n}\right)_{x}-l k_{0}\left(w_{n x}-l \varphi_{n}\right)\right] \rightarrow 0 \text { in } L^{2}(0,1) \\
\lambda_{n}^{4}\left[i \lambda_{n} \psi_{n}-\tilde{\psi}_{n}\right] \rightarrow 0 \text { in } \tilde{H}_{*}^{1}(0,1) \\
\lambda_{n}^{4}\left[i \rho_{2} \lambda_{n} \tilde{\psi}_{n}-b \psi_{n x x}+k\left(\varphi_{n x}+\psi_{n}+l w_{n}\right)\right] \rightarrow 0 \text { in } L^{2}(0,1) \\
\lambda_{n}^{4}\left[i \lambda_{n} w_{n}-\tilde{w}_{n}\right] \rightarrow 0 \text { in } \tilde{H}_{*}^{1}(0,1) \\
\lambda_{n}^{4}\left[i \rho_{1} \lambda_{n} \tilde{w}_{n}-k_{0}\left(w_{n x}-l \varphi_{n}\right)_{x}+l k\left(\varphi_{n x}+\psi_{n}+l w_{n}\right)+\delta \theta_{n x}\right] \rightarrow 0 \text { in } L^{2}(0,1) \\
\lambda_{n}^{4}\left[i \rho_{3} \lambda_{n} \theta_{n}-\beta \theta_{n x x}+\delta \tilde{w}_{n x}\right] \rightarrow 0 \text { in } L^{2}(0,1)
\end{array}\right.
$$

Estimates on $\theta_{n x}$ and $\theta_{n}$ Taking the inner product of $\lambda_{n}^{4}\left(i \lambda_{n} I-\mathcal{A}\right) \Phi_{n}$ with $\Phi_{n}$ in $\mathcal{H}$ and using (17), we get

$$
\operatorname{Re}\left\langle\lambda_{n}^{4}\left(i \lambda_{n} I-\mathcal{A}\right) \Phi_{n}, \Phi_{n}\right\rangle_{\mathcal{H}}=\operatorname{Re}\left(i \lambda_{n}^{5}\left\|\Phi_{n}\right\|_{L^{2}(0,1)}^{2}+\beta \lambda_{n}^{4}\left\|\theta_{n x}\right\|_{L^{2}(0,1)}^{2}\right)=\beta \lambda_{n}^{4}\left\|\theta_{n x}\right\|_{L^{2}(0,1)}^{2} .
$$

So (46) and (105) imply that

$$
\lambda_{n}^{2} \theta_{n x} \longrightarrow 0 \quad \text { in } L^{2}(0,1) .
$$

Because $\theta_{n}$ in $H_{*}^{1}(0,1)$ and thanks to Poincaré's inequality, we deduce that

$$
\lambda_{n}^{2} \theta_{n} \longrightarrow 0 \text { in } L^{2}(0,1)
$$

Estimates on $\varphi_{n}, \psi_{n}$ and $w_{n}$ Multiplying $(106)_{1},(106)_{3}$ and (106) $)_{5}$ by $\frac{1}{\lambda_{n}^{5}}$, and using (46) and (47), we obtain (54).

Estimate on $\frac{1}{\lambda_{n}} w_{n x x}$ Multiplying (106) 6 by $\frac{1}{\lambda_{n}^{5}}$ and using (46), (47) and (107), we conclude (55).

Estimates on $\lambda_{n} w_{n x}, \lambda_{n} w_{n}, \tilde{w}_{n x}$ and $\tilde{w}_{n}$ Taking the inner product of $(106)_{7}$ with $\frac{i}{\lambda_{n}^{3}} w_{n x}$ in $L^{2}(0,1)$ and using (46) and (47), we get

$$
\begin{aligned}
& \rho_{3}\left\langle\lambda_{n}^{2} \theta_{n}, w_{n x}\right\rangle_{L^{2}(0,1)}-\beta\left\langle\lambda_{n} \theta_{n x x}, i w_{n x}\right\rangle_{L^{2}(0,1)} \\
& \quad-\delta\left\langle\lambda_{n}\left(i \lambda_{n} w_{n x}-\tilde{w}_{n x}\right), i w_{n x}\right\rangle_{L^{2}(0,1)}+\delta \lambda_{n}^{2}\left\|w_{n x}\right\|_{L^{2}(0,1)}^{2} \longrightarrow 0,
\end{aligned}
$$

then, integrating by parts and using the boundary conditions, we deduce that

$$
\begin{aligned}
& \rho_{3}\left\langle\lambda_{n}^{2} \theta_{n}, w_{n x}\right\rangle_{L^{2}(0,1)}+\beta\left\langle\lambda_{n}^{2} \theta_{n x}, \frac{i}{\lambda_{n}} w_{n x x}\right\rangle_{L^{2}(0,1)} \\
& \quad-\delta\left\langle\lambda_{n}\left(i \lambda_{n} w_{n x}-\tilde{w}_{n x}\right), i w_{n x}\right\rangle_{L^{2}(0,1)}+\delta \lambda_{n}^{2}\left\|w_{n x}\right\|_{L^{2}(0,1)}^{2} \longrightarrow 0 .
\end{aligned}
$$

Combining (46), (47), (55), (106)5, (107) and (108), we get

$$
\lambda_{n} w_{n x} \longrightarrow 0 \text { in } L^{2}(0,1) .
$$


Moreover, again by multiplying (106) 5 by $\frac{1}{\lambda_{n}^{4}}$, we find

$$
\tilde{w}_{n x} \rightarrow 0 \text { in } L^{2}(0,1)
$$

and, as $w_{n}, \tilde{w}_{n} \in \tilde{H}_{*}^{1}(0,1)$ and thanks to Poincaré's inequality, we have also (59) and (60).

Estimates on $\lambda_{n}^{2} w_{n}$ and $\lambda_{n} \widetilde{w}_{n}$ Multiplying (106) $)_{1}$ and (106) 3 by $\frac{1}{\lambda_{n}^{4}}$, and using (46) and (47), we have

$$
\left(\lambda_{n} \varphi_{n}\right)_{n} \text { and }\left(\lambda_{n} \psi_{n}\right)_{n} \text { are bounded in } L^{2}(0,1)
$$

Taking the inner product of $(106)_{6}$ with $\frac{i}{\lambda_{n}^{3}} \widetilde{w}_{n}$ in $L^{2}(0,1)$, integrating by parts and using (46), (47) and the boundary conditions, we get

$$
\begin{aligned}
& \rho_{1}\left\|\lambda_{n} \tilde{w}_{n}\right\|_{L^{2}(0,1)}^{2}+k_{0}\left\langle\lambda_{n}\left(w_{n x}-l \varphi_{n}\right), i \tilde{w}_{n x}\right\rangle_{L^{2}(0,1)} \\
& \quad+l k\left\langle\lambda_{n}\left(\varphi_{n x}+\psi_{n}+l w_{n}\right), i \tilde{w}_{n}\right\rangle_{L^{2}(0,1)}+\delta\left\langle\lambda_{n} \theta_{n x}, i \tilde{w}_{n}\right\rangle_{L^{2}(0,1)} \rightarrow 0 .
\end{aligned}
$$

So, using (59), (60), (107), (110), (111) and (112), we deduce that

$$
\lambda_{n} \widetilde{w}_{n} \longrightarrow 0 \text { in } L^{2}(0,1),
$$

and by multiplying (106) 5 by $\frac{1}{\lambda_{n}^{3}}$ and using (47), we find

$$
\lambda_{n}^{2} w_{n} \longrightarrow 0 \text { in } L^{2}(0,1)
$$

Estimate on $\varphi_{n x}$ Multiplying (106) 2 and (106) 4 by $\frac{1}{\lambda_{n}^{5}}$ and using (46) and (47), we get

$$
\left(\frac{1}{\lambda_{n}} \varphi_{n x x}\right)_{n} \text { and }\left(\frac{1}{\lambda_{n}} \psi_{n x x}\right)_{n} \text { are bounded in } L^{2}(0,1) \text {. }
$$

On the other hand, taking the inner product of $(106)_{6}$ with $\frac{1}{\lambda_{n}^{4}} \varphi_{n x}$ in $L^{2}(0,1)$, integrating by parts and using (46), (47) and the boundary conditions, we get

$$
\begin{aligned}
& i \rho_{1}\left\langle\lambda_{n} \tilde{w}_{n}, \varphi_{n x}\right\rangle_{L^{2}(0,1)}+\left\langle l k\left(\psi_{n}+l w_{n}\right)+\delta \theta_{n x}, \varphi_{n x}\right\rangle_{L^{2}(0,1)} \\
& +l\left(k+k_{0}\right)\left\|\varphi_{n x}\right\|_{L^{2}(0,1)}^{2}+k_{0}\left\langle\lambda_{n} w_{n x}, \frac{1}{\lambda_{n}} \varphi_{n x x}\right\rangle_{L^{2}(0,1)} \rightarrow 0 .
\end{aligned}
$$

Then, using (54), (107), (110), (114) and (116), we deduce that

$$
\varphi_{n x} \rightarrow 0 \text { in } L^{2}(0,1) \text {. }
$$

Estimates on $\lambda_{n} \varphi_{n}$ and $\tilde{\varphi}_{n}$ Taking the inner product of $(106)_{2}$ with $\frac{1}{\lambda_{n}^{4}} \varphi_{n}$ in $L^{2}(0,1)$, using (46) and (47), integrating by parts and using the boundary conditions, we obtain

$$
\begin{aligned}
- & \rho_{1}\left\langle\tilde{\varphi}_{n},\left(i \lambda_{n} \varphi_{n}-\tilde{\varphi}_{n}\right)\right\rangle_{L^{2}(0,1)}-\rho_{1}\left\|\tilde{\varphi}_{n}\right\|_{L^{2}(0,1)}^{2} \\
& +k\left\langle\left(\varphi_{n x}+\psi_{n}+l w_{n}\right), \varphi_{n x}\right\rangle_{L^{2}(0,1)}-l k_{0}\left\langle\left(w_{n x}-l \varphi_{n}\right), \varphi_{n}\right\rangle_{L^{2}(0,1)} \rightarrow 0,
\end{aligned}
$$

then, using (54), (106) 1 and (118), we find

$$
\tilde{\varphi}_{n} \rightarrow 0 \text { in } L^{2}(0,1) .
$$


Moreover, multiplying (106) $)_{1}$ by $\frac{1}{\lambda_{n}^{4}}$ and using (47) and (119), we get

$$
\lambda_{n} \varphi_{n} \rightarrow 0 \text { in } L^{2}(0,1)
$$

Estimates on $\psi_{n x}$ and $\tilde{\psi}_{n}$ and conclusion First, taking the inner product of (106) 4 with $\frac{1}{\lambda_{n}^{4}} \psi_{n}$ in $L^{2}(0,1)$, using (46) and (47), integrating by parts and using the boundary conditions, we obtain

$$
\begin{aligned}
& -\rho_{2}\left\langle\tilde{\psi}_{n},\left(i \lambda_{n} \psi_{n}-\tilde{\psi}_{n}\right)\right\rangle_{L^{2}(0,1)}-\rho_{2}\left\|\tilde{\psi}_{n}\right\|_{L^{2}(0,1)}^{2} \\
& +b\left\|\psi_{n x}\right\|_{L^{2}(0,1)}^{2}+k\left\langle\left(\varphi_{n x}+\psi_{n}+l w_{n}\right), \psi_{n}\right\rangle_{L^{2}(0,1)} \rightarrow 0
\end{aligned}
$$

then, using (54) and (106) 3 , we find

$$
b\left\|\psi_{n x}\right\|_{L^{2}(0,1)}^{2}-\rho_{2}\left\|\tilde{\psi}_{n}\right\|_{L^{2}(0,1)}^{2} \rightarrow 0 .
$$

Second, taking the inner product of $(106)_{2}$ with $\frac{1}{\lambda_{n}^{4}} \psi_{n x}$, integrating by parts and using the boundary conditions, (46) and (47), we obtain

$$
\begin{aligned}
& -k\left\|\psi_{n x}\right\|_{L^{2}(0,1)}^{2}+k\left\langle\varphi_{n x}, \psi_{n x x}\right\rangle_{L^{2}(0,1)}+i \rho_{1} \lambda_{n}\left\langle\tilde{\varphi}_{n}, \psi_{n x}\right\rangle_{L^{2}(0,1)} \\
& \quad-l\left(k+k_{0}\right)\left\langle w_{n x}, \psi_{n x}\right\rangle_{L^{2}(0,1)}-l^{2} k_{0}\left\langle\varphi_{n x}, \psi_{n}\right\rangle_{L^{2}(0,1)} \rightarrow 0 \text { in } L^{2}(0,1) .
\end{aligned}
$$

Exploiting (110) and (118), we get

$$
-k\left\|\psi_{n x}\right\|_{L^{2}(0,1)}^{2}+k\left\langle\varphi_{n x}, \psi_{n x x}\right\rangle_{L^{2}(0,1)}+i \rho_{1} \lambda_{n}\left\langle\tilde{\varphi}_{n}, \psi_{n x}\right\rangle_{L^{2}(0,1)} \rightarrow 0 \text { in } L^{2}(0,1) .
$$

Third, taking the inner product of $\frac{k}{b \lambda_{n}^{4}} \varphi_{n x}$ with $(106)_{4}$, integrating by parts and using the boundary conditions, (46) and (47), we obtain

$$
\begin{aligned}
& -k\left\langle\varphi_{n x}, \psi_{n x x}\right\rangle_{L^{2}(0,1)}-\frac{i \rho_{2} k \lambda_{n}}{b}\left\langle\tilde{\varphi}_{n}, \psi_{n x}\right\rangle_{L^{2}(0,1)}+\frac{k^{2}}{b}\left\langle\varphi_{n x},\left(\varphi_{n x}+\psi_{n}+l w_{n}\right)\right\rangle_{L^{2}(0,1)} \\
& +\frac{k \rho_{2}}{b}\left\langle\varphi_{n}, i \lambda_{n}\left(i \lambda_{n} \psi_{n x}-\tilde{\psi}_{n x}\right)\right\rangle_{L^{2}(0,1)}-\frac{i k \rho_{2}}{b}\left\langle\lambda_{n}\left(i \lambda_{n} \varphi_{n}-\tilde{\varphi}_{n}\right), \psi_{n x}\right\rangle_{L^{2}(0,1)} \rightarrow 0 \text { in } L^{2}(0,1),
\end{aligned}
$$

so, from $(106)_{1},(106)_{3}$ and (118), we find

$$
-k\left\langle\varphi_{n x}, \psi_{n x x}\right\rangle_{L^{2}(0,1)}-\frac{i \rho_{2} k \lambda_{n}}{b}\left\langle\tilde{\varphi}_{n}, \psi_{n x}\right\rangle_{L^{2}(0,1)} \rightarrow 0 \text { in } L^{2}(0,1) .
$$

By adding (122) and (123) and using the equality $\rho_{1} b-\rho_{2} k=0$, we see that

$$
\psi_{n x} \rightarrow 0 \text { in } L^{2}(0,1) .
$$

Therefore, from (121), we get

$$
\widetilde{\psi}_{n} \rightarrow 0 \text { in } L^{2}(0,1) .
$$

Finally, the limits (54), (59), (108), (110), (118), (119), (124) and (125) imply (50), which is a contradiction with (46). Consequently, (103) with $j=4$ holds. 
4.2 Case of System (1) with $\rho_{1} b-\rho_{2} k \neq 0$

The limit (105) with $j=10$ implies (106) with $\lambda_{n}^{10}$ instead of $\lambda_{n}^{4}$; that is

$$
\left\{\begin{array}{l}
\lambda_{n}^{10}\left[i \lambda_{n} \varphi_{n}-\tilde{\varphi}_{n}\right] \rightarrow 0 \text { in } H_{*}^{1}(0,1) \\
\lambda_{n}^{10}\left[i \rho_{1} \lambda_{n} \tilde{\varphi}_{n}-k\left(\varphi_{n x}+\psi_{n}+l w_{n}\right)_{x}-l k_{0}\left(w_{n x}-l \varphi_{n}\right)\right] \rightarrow 0 \text { in } L^{2}(0,1), \\
\lambda_{n}^{10}\left[i \lambda_{n} \psi_{n}-\tilde{\psi}_{n}\right] \rightarrow 0 \text { in } \tilde{H}_{*}^{1}(0,1) \\
\lambda_{n}^{10}\left[i \rho_{2} \lambda_{n} \tilde{\psi}_{n}-b \psi_{n x x}+k\left(\varphi_{n x}+\psi_{n}+l w_{n}\right)\right] \rightarrow 0 \text { in } L^{2}(0,1), \\
\lambda_{n}^{10}\left[i \lambda_{n} w_{n}-\tilde{w}_{n}\right] \rightarrow 0 \text { in } \tilde{H}_{*}^{1}(0,1) \\
\lambda_{n}^{10}\left[i \rho_{1} \lambda_{n} \tilde{w}_{n}-k_{0}\left(w_{n x}-l \varphi_{n}\right)_{x}+l k\left(\varphi_{n x}+\psi_{n}+l w_{n}\right)+\delta \theta_{n x}\right] \rightarrow 0 \text { in } L^{2}(0,1), \\
\lambda_{n}^{10}\left[i \rho_{3} \lambda_{n} \theta_{n}-\beta \theta_{n x x}+\delta \tilde{w}_{n x}\right] \rightarrow 0 \text { in } L^{2}(0,1) .
\end{array}\right.
$$

Similarly to the case $\rho_{1} b-\rho_{2} k=0$ (Sect. 4.1), we see that (54), (55), (112), (116) and (118) hold (for (112) and (118), we have just to use $\frac{1}{\lambda_{n}^{10}}$ instead of $\frac{1}{\lambda_{n}^{4}}$, and for (116), we use $\frac{1}{\lambda_{n}^{11}}$ instead of $\frac{1}{\lambda_{n}^{5}}$ ).

Moreover, the same computations as in Sect. 4.1 (case $\rho_{1} b-\rho_{2} k=0$ ) give (instead of (107), (108), (110), (111), (59) and (60))

$$
\lambda_{n}^{5} \theta_{n x}, \lambda_{n}^{5} \theta_{n},\left|\lambda_{n}\right|^{\frac{5}{2}} w_{n x},\left|\lambda_{n}\right|^{\frac{3}{2}} \widetilde{w}_{n x},\left|\lambda_{n}\right|^{\frac{3}{2}} \tilde{w}_{n},\left|\lambda_{n}\right|^{\frac{5}{2}} w_{n} \longrightarrow 0 \text { in } L^{2}(0,1)
$$

(for (110), we replace $\frac{i}{\lambda_{n}^{3}} w_{n x}$ by $\frac{i}{\lambda_{n}^{6}} w_{n x}$ and use (55), and for (111), we use $\frac{1}{\left|\lambda_{n}\right|^{\frac{17}{2}}}$ instead of $\frac{1}{\lambda_{n}^{4}}$ ). Now, we prove some other limits to get (50).

Estimate on $w_{n x x}$ Dividing (126) 6 by $\lambda_{n}^{10}$ and using (46), (47) and (127), we deduce that

$$
\left(w_{n x x}\right)_{n} \text { is uniformly bounded in } L^{2}(0,1) \text {. }
$$

Estimates on $\varphi_{n x}, \varphi_{n}$ and $\tilde{\varphi}_{n}$ Taking the inner product of (126) 6 with $\frac{\varphi_{n x}}{\lambda_{n}^{9}}$ in $L^{2}(0,1)$, integrating by parts and using (46), (47) and the boundary conditions, we get

$$
\begin{aligned}
- & \rho_{1}\left\langle\tilde{w}_{n}, \lambda_{n}\left(i \lambda_{n} \varphi_{n x}-\tilde{\varphi}_{n x}\right)\right\rangle_{L^{2}(0,1)}+\rho_{1}\left\langle\lambda_{n} \tilde{w}_{n x}, \tilde{\varphi}_{n}\right\rangle_{L^{2}(0,1)} \\
& +k_{0}\left\langle\lambda_{n}^{2} w_{n x}, \frac{\varphi_{n x x}}{\lambda_{n}}\right\rangle_{L^{2}(0,1)}+l\left(k+k_{0}\right) \lambda_{n}\left\|\varphi_{n x}\right\|_{L^{2}(0,1)}^{2} \\
& +l k\left\langle\lambda_{n}\left(\psi_{n}+l w_{n}\right), \varphi_{n x}\right\rangle_{L^{2}(0,1)}+\delta\left\langle\lambda_{n} \theta_{n x}, \varphi_{n x}\right\rangle_{L^{2}(0,1)} \longrightarrow 0,
\end{aligned}
$$

hence, using (126) 1 , (112), (116), (118) and (127), we obtain

$$
\left|\lambda_{n}\right|^{\frac{1}{2}} \varphi_{n x} \longrightarrow 0 \text { in } L^{2}(0,1) .
$$

Therefore, according to Poincaré's inequality, (129) leads to

$$
\left|\lambda_{n}\right|^{\frac{1}{2}} \varphi_{n} \longrightarrow 0 \text { in } L^{2}(0,1) .
$$

On the other hand, taking the inner product of $(126)_{2}$ with $\frac{\varphi_{n}}{\lambda_{n}^{9}}$ in $L^{2}(0,1)$, integrating by parts and using (46), (47) and the boundary conditions, we get

$$
\begin{aligned}
- & \rho_{1} \lambda_{n}\left\langle\tilde{\varphi}_{n},\left(i \lambda_{n} \varphi_{n}-\tilde{\varphi}_{n}\right)\right\rangle_{L^{2}(0,1)}-\rho_{1} \lambda_{n}\left\|\tilde{\varphi}_{n}\right\|_{L^{2}(0,1)}^{2} \\
& +k \lambda_{n}\left\langle\left(\varphi_{n x}+\psi_{n}+l w_{n}\right), \varphi_{n x}\right\rangle_{L^{2}(0,1)}-l k_{0} \lambda_{n}\left\langle\left(w_{n x}-l \varphi_{n}\right), \varphi_{n}\right\rangle_{L^{2}(0,1)} \longrightarrow 0,
\end{aligned}
$$


this implies

$$
\begin{aligned}
& -\rho_{1}\left\{\tilde{\varphi}_{n}, \lambda_{n}\left(i \lambda_{n} \varphi_{n}-\tilde{\varphi}_{n}\right)\right\rangle_{L^{2}(0,1)}-\rho_{1} \lambda_{n}\left\|\tilde{\varphi}_{n}\right\|_{L^{2}(0,1)}^{2}+k \lambda_{n}\left\|\varphi_{n x}\right\|_{L^{2}(0,1)}^{2} \\
& \quad+k\left\langle\left(\lambda_{n} \psi_{n}+l \lambda_{n} w_{n}\right), \varphi_{n x}\right\rangle_{L^{2}(0,1)}-l k_{0}\left\langle\left(\lambda_{n} w_{n x}-l \lambda_{n} \varphi_{n}\right), \varphi_{n}\right\rangle_{L^{2}(0,1)} \longrightarrow 0,
\end{aligned}
$$

so, using (126) $)_{1},(112),(127)$ and (129), we deduce that

$$
\left|\lambda_{n}\right|^{\frac{1}{2}} \tilde{\varphi}_{n} \longrightarrow 0 \text { in } L^{2}(0,1)
$$

and from $(126)_{1}$, we obtain that

$$
\left|\lambda_{n}\right|^{\frac{3}{2}} \varphi_{n} \longrightarrow 0 \text { in } L^{2}(0,1)
$$

Estimates on $\lambda_{n} \varphi_{n x}$ and $\lambda_{n} \tilde{\varphi}_{n}$ Multiplying (126) 2 by $\frac{1}{\left|\lambda_{n}\right|^{10+\frac{1}{2}}}$ and using (47), we get

$$
i \rho_{1} \frac{\lambda_{n}}{\left|\lambda_{n}\right|^{\frac{1}{2}}} \tilde{\varphi}_{n}-k \frac{\varphi_{n x x}}{\left|\lambda_{n}\right|^{\frac{1}{2}}}-k \frac{\psi_{n x}}{\left|\lambda_{n}\right|^{\frac{1}{2}}}-l\left(k+k_{0}\right) \frac{w_{n x}}{\left|\lambda_{n}\right|^{\frac{1}{2}}}+l^{2} k_{0} \frac{\varphi_{n}}{\left|\lambda_{n}\right|^{\frac{1}{2}}} \longrightarrow 0 \text { in } L^{2}(0,1),
$$

then, using (46) and (131), we deduce that

$$
\frac{\varphi_{n x x}}{\left|\lambda_{n}\right|^{\frac{1}{2}}} \longrightarrow 0 \text { in } L^{2}(0,1) \text {. }
$$

On the other hand, by integrating by parts and using the boundary conditions, we see that

$$
\begin{aligned}
\lambda_{n}\left\langle w_{n x x}, i \lambda_{n} \varphi_{n x}\right\rangle_{L^{2}(0,1)} & =\lambda_{n}^{2}\left\langle i w_{n x}, \varphi_{n x x}\right\rangle_{L^{2}(0,1)} \\
& =\left\langle\lambda_{n}\left(i \lambda_{n} w_{n x}-\tilde{w}_{n x}\right), \varphi_{n x x}\right\rangle_{L^{2}(0,1)}+\lambda_{n}\left\langle\tilde{w}_{n x}, \varphi_{n x x}\right\rangle_{L^{2}(0,1)} \\
& =\left\langle\lambda_{n}^{2}\left(i \lambda_{n} w_{n x}-\tilde{w}_{n x}\right), \frac{\varphi_{n x x}}{\lambda_{n}}\right\rangle_{L^{2}(0,1)}+\left\langle\lambda_{n}\left|\lambda_{n}\right|^{\frac{1}{2}} \tilde{w}_{n x}, \frac{\varphi_{n x x}}{\left|\lambda_{n}\right|^{\frac{1}{2}}}\right\rangle_{L^{2}(0,1)},
\end{aligned}
$$

then, using (47), (126), , (127) and (133), we obtain

$$
\lambda_{n}\left\langle w_{n x x}, i \lambda_{n} \varphi_{n x}\right\rangle_{L^{2}(0,1)} \longrightarrow 0 .
$$

Furthermore, integrating by parts and using the boundary conditions, we have

$$
\begin{aligned}
\lambda_{n}\left\langle\left(\varphi_{n x}+\psi_{n}+l w_{n}\right)_{x}, \tilde{\varphi}_{n}\right\rangle_{L^{2}(0,1)}= & -\lambda_{n}\left\langle\left(\varphi_{n x}+\psi_{n}+l w_{n}\right), \tilde{\varphi}_{n x}\right\rangle_{L^{2}(0,1)} \\
= & -\frac{1}{l k}\left\langle\lambda _ { n } ^ { 2 } \left[ i \lambda_{n} \rho_{1} \tilde{w}_{n}-k_{0}\left(w_{n x}-l \varphi_{n}\right)_{x}\right.\right. \\
& \left.\left.+l k\left(\varphi_{n x}+\psi_{n}+l w_{n}\right)+\delta \theta_{n x}\right], \frac{\tilde{\varphi}_{n x}}{\lambda_{n}}\right\rangle_{L^{2}(0,1)} \\
& -\frac{1}{l k}\left\langle\left(i \lambda_{n} \rho_{1} \tilde{w}_{n}+\delta \theta_{n x}\right), \lambda_{n}\left(i \lambda_{n} \varphi_{n x}-\tilde{\varphi}_{n x}\right)\right\rangle_{L^{2}(0,1)} \\
& +\frac{k_{0}}{l k}\left\langle\left(w_{n x}-l \varphi_{n}\right)_{x}, \lambda_{n}\left(i \lambda_{n} \varphi_{n x}-\tilde{\varphi}_{n x}\right)\right\rangle_{L^{2}(0,1)}-\frac{\lambda_{n}^{3}}{l k}\left\langle i \rho_{1} \tilde{w}_{n x}, i \varphi_{n}\right\rangle_{L^{2}(0,1)} \\
& +\frac{\delta}{l k}\left\langle\lambda_{n}^{2} \theta_{n x}, i \varphi_{n x}\right\rangle_{L^{2}(0,1)}-\frac{k_{0} \lambda_{n}}{l k}\left\langle w_{n x x}, i \lambda_{n} \varphi_{n x}\right\rangle_{L^{2}(0,1)}-\frac{k_{0} \lambda_{n}^{2}}{k} i\left\|\varphi_{n x}\right\|_{L^{2}(0,1)}^{2},
\end{aligned}
$$

then, using $(126)_{1},(126)_{6},(127),(128),(132)$ and (134), we find

$$
\lambda_{n}\left\langle\left(\varphi_{n x}+\psi_{n}+l w_{n}\right)_{x}, \tilde{\varphi}_{n}\right\rangle_{L^{2}(0,1)}+\frac{k_{0}}{k} i\left\|\lambda_{n} \varphi_{n x}\right\|_{L^{2}(0,1)}^{2} \longrightarrow 0 .
$$


Taking the inner product of (126) 2 with $\frac{\tilde{\varphi}_{n}}{\lambda_{n}^{9}}$ in $L^{2}(0,1)$ and using (46) and (47s), we get

$$
\rho_{1} i\left\|\lambda_{n} \tilde{\varphi}_{n}\right\|_{L^{2}(0,1)}^{2}-k \lambda_{n}\left\langle\left(\varphi_{n x}+\psi_{n}+l w_{n}\right)_{x}, \tilde{\varphi}_{n}\right\rangle_{L^{2}(0,1)}-l k_{0}\left\langle\left(\lambda_{n} w_{n x}-l \lambda_{n} \varphi_{n}\right), \tilde{\varphi}_{n}\right\rangle_{L^{2}(0,1)} \longrightarrow 0
$$

then, using (135), we obtain

$$
\rho_{1} i\left\|\lambda_{n} \tilde{\varphi}_{n}\right\|_{L^{2}(0,1)}^{2}+i k_{0}\left\|\lambda_{n} \varphi_{n x}\right\|_{L^{2}(0,1)}^{2}-l k_{0}\left\langle\left(\lambda_{n} w_{n x}-l \lambda_{n} \varphi_{n}\right), \tilde{\varphi}_{n}\right\rangle_{L^{2}(0,1)} \longrightarrow 0
$$

and from (127), (131) and (132), we deduce that

$$
\lambda_{n} \tilde{\varphi}_{n} \longrightarrow 0 \text { in } L^{2}(0,1)
$$

and

$$
\lambda_{n} \varphi_{n x} \longrightarrow 0 \text { in } L^{2}(0,1)
$$

Estimates on $\psi_{n x}$ and $\tilde{\psi}_{n}$ and conclusion Taking the inner product of $(126)_{2}$ with $\frac{\psi_{n x}}{\lambda_{n}^{10}}$ in $L^{2}(0,1)$ and using (46) and (47), we get

$$
\begin{gathered}
\rho_{1}\left\langle i \lambda_{n} \tilde{\varphi}_{n}, \psi_{n x}\right\rangle_{L^{2}(0,1)}-k\left\langle\varphi_{n x x}, \psi_{n x}\right\rangle_{L^{2}(0,1)}-k\left\|\psi_{n x}\right\|_{L^{2}(0,1)}^{2} \\
-l\left(k+k_{0}\right)\left\langle w_{n x}, \psi_{n x}\right\rangle_{L^{2}(0,1)}+l^{2} k_{0}\left\langle\varphi_{n}, \psi_{n x}\right\rangle_{L^{2}(0,1)} \rightarrow 0
\end{gathered}
$$

then, integrating by parts and using the boundary conditions, we obtain

$$
\begin{gathered}
\rho_{1}\left\langle i \lambda_{n} \tilde{\varphi}_{n}, \psi_{n x}\right\rangle_{L^{2}(0,1)}+k\left\langle\lambda_{n} \varphi_{n x}, \frac{\psi_{n x x}}{\lambda_{n}}\right\rangle_{L^{2}(0,1)}-k\left\|\psi_{n x}\right\|_{L^{2}(0,1)}^{2} \\
-l\left(k+k_{0}\right)\left\langle w_{n x}, \psi_{n x}\right\rangle_{L^{2}(0,1)}+l^{2} k_{0}\left\langle\varphi_{n}, \psi_{n x}\right\rangle_{L^{2}(0,1)} \rightarrow 0,
\end{gathered}
$$

so, using (54), (116), (127), (136) and (137), we deduce that

$$
\psi_{n x} \longrightarrow 0 \text { in } L^{2}(0,1)
$$

Taking the inner product of $(126)_{4}$ with $\frac{\psi_{n}}{\lambda_{n}^{10}}$ in $L^{2}(0,1)$, integrating by parts and using (46), (47) and the boundary conditions, we get

$$
\begin{aligned}
& -\rho_{2}\left\langle\tilde{\psi}_{n},\left(i \lambda_{n} \psi_{n}-\tilde{\psi}_{n}\right)\right\rangle_{L^{2}(0,1)}-\rho_{2}\left\|\tilde{\psi}_{n}\right\|_{L^{2}(0,1)}^{2}+b\left\|\psi_{n x}\right\|_{L^{2}(0,1)}^{2} \\
& +\left\langle k\left(\varphi_{n x}+\psi_{n}+l w_{n}\right), \psi_{n}\right\rangle_{L^{2}(0,1)} \longrightarrow 0
\end{aligned}
$$

hence, using (54), (126) 3 and (138), we get

$$
\tilde{\psi}_{n} \longrightarrow 0 \text { in } L^{2}(0,1)
$$

A combination of the limits (54), (118), (127), (136), (138) and (139) leads to (50), which is a contradiction with (46). Consequently, (103) with $j=10$ holds. 
4.3 Case of System (4) with $\rho_{1} b-\rho_{2} k=0$

The limit (105) with $j=4$ implies that

$$
\left\{\begin{array}{l}
\lambda_{n}^{4}\left[i \lambda_{n} \varphi_{n}-\tilde{\varphi}_{n}\right] \rightarrow 0 \text { in } H_{*}^{1}(0,1), \\
\lambda_{n}^{4}\left[i \rho_{1} \lambda_{n} \tilde{\varphi}_{n}-k\left(\varphi_{n x}+\psi_{n}+l w_{n}\right)_{x}-l k_{0}\left(w_{n x}-l \varphi_{n}\right)\right] \rightarrow 0 \text { in } L^{2}(0,1), \\
\lambda_{n}^{4}\left[i \lambda_{n} \psi_{n}-\tilde{\psi}_{n}\right] \rightarrow 0 \text { in } \tilde{H}_{*}^{1}(0,1), \\
\lambda_{n}^{4}\left[i \rho_{2} \lambda_{n} \tilde{\psi}_{n}-b \psi_{n x x}+k\left(\varphi_{n x}+\psi_{n}+l w_{n}\right)\right] \rightarrow 0 \text { in } L^{2}(0,1), \\
\lambda_{n}^{4}\left[i \lambda_{n} w_{n}-\tilde{w}_{n}\right] \rightarrow 0 \text { in } \tilde{H}_{*}^{1}(0,1), \\
\lambda_{n}^{4}\left[i \rho_{1} \lambda_{n} \tilde{w}_{n}-k_{0}\left(w_{n x}-l \varphi_{n}\right)_{x}+l k\left(\varphi_{n x}+\psi_{n}+l w_{n}\right)+\delta \tilde{\theta}_{n x}\right] \rightarrow 0 \text { in } L^{2}(0,1), \\
\lambda_{n}^{4}\left[i \lambda_{n} \theta_{n}-\tilde{\theta}_{n}\right] \rightarrow 0 \text { in } \tilde{H}_{*}^{1}(0,1), \\
\lambda_{n}^{4}\left[i \rho_{3} \lambda_{n} \theta_{n}-\beta\left(\theta_{n}+\gamma \tilde{\theta}_{n}\right)_{x x}+\delta \tilde{w}_{n x}\right] \rightarrow 0 \text { in } L^{2}(0,1) .
\end{array}\right.
$$

Estimates on $\theta_{n x}, \theta_{n}, \tilde{\theta}_{n x}$ and $\tilde{\theta}_{n}$ and conclusion Taking the inner product of $\lambda_{n}^{4}\left(i \lambda_{n} I-\mathcal{A}\right) \Phi_{n}$ with $\Phi_{n}$ in $\mathcal{H}$ and using (17), we get

$$
\begin{aligned}
\operatorname{Re}\left\langle\lambda_{n}^{4}\left(i \lambda_{n} I-\mathcal{A}\right) \Phi_{n}, \Phi_{n}\right\rangle_{\mathcal{H}} & =\operatorname{Re}\left(i \lambda_{n}^{5}\left\|\Phi_{n}\right\|_{L^{2}(0,1)}^{2}+\gamma \lambda_{n}^{4}\left\|\tilde{\theta}_{n x}\right\|_{L^{2}(0,1)}^{2}\right) \\
& =\gamma \lambda_{n}^{4}\left\|\tilde{\theta}_{n x}\right\|_{L^{2}(0,1)}^{2} .
\end{aligned}
$$

So (46) and (105) imply that

$$
\lambda_{n}^{2} \tilde{\theta}_{n x} \longrightarrow 0 \text { in } L^{2}(0,1) .
$$

Because $\theta_{n}$ in $H_{*}^{1}(0,1)$ and thanks to Poincaré's inequality, we deduce that

$$
\lambda_{n}^{2} \tilde{\theta}_{n} \longrightarrow 0 \text { in } L^{2}(0,1)
$$

Multiplying (140) 7 by $\frac{1}{\lambda_{n}^{2}}$ and using (46), (47), (141) and (142), we have

$$
\lambda_{n}^{3} \theta_{n x} \longrightarrow 0 \quad \text { in } L^{2}(0,1)
$$

and

$$
\lambda_{n}^{3} \theta_{n} \longrightarrow 0 \quad \text { in } L^{2}(0,1),
$$

so (107) and (108) hold. Consequently, the proof can be ended exactly as in case of System (1) with $j=4$ (Sect. 4.1). 


\subsection{Case of System (4) with $\rho_{1} b-\rho_{2} k \neq 0$}

The limit (105) with $j=10$ implies (140) with $\lambda_{n}^{10}$ instead of $\lambda_{n}^{4}$. Similar calculations as in the case of system (1) with $\rho_{1} b-\rho_{2} k \neq 0$ (Sect. 4.2) give the desired result. We omit the details.

Hence, the proof of our Theorem 4.1 is completed.

Acknowledgements The author would like to express his gratitude to the anonymous referee for very careful reading and punctual suggestions.

Open Access This article is distributed under the terms of the Creative Commons Attribution 4.0 International License (http:// creativecommons.org/licenses/by/4.0/), which permits unrestricted use, distribution, and reproduction in any medium, provided you give appropriate credit to the original author(s) and the source, provide a link to the Creative Commons license, and indicate if changes were made.

\section{References}

1. Afilal, M.; Guesmia,A.; Soufyane, A.: On the uniform stability for a linear thermoelastic Bresse system with second sound (2018) (submitted)

2. Bresse, J.A.C.: Cours de Mécanique Appliquée. Mallet Bachelier, Paris (1859)

3. Chandrasekharaiah, D.: Hyberpolic thermoelasticity: a review of recent literature. Appl. Mech. Rev. 51, 705-729 (1998)

4. Dell'Oro, F.: Asymptotic stability of thermoelastic systems of Bresse type. J. Differ. Equations 258, 3902-3927 (2015)

5. Fatori, L.H.; Rivera, J.M.: Rates of decay to weak thermoelastic Bresse system. IMA J. Appl. Math. 75, 881-904 (2010)

6. Green, A.; Naghdi, P.: A re-examination of the basic postulates of thermomechanics. Proc. R. Soc. Lond. A 432, 171-194 (1991)

7. Green, A.; Naghdi, P.: On undamped heat waves in an elastic solid. J. Therm. Stresses 15, 253-264 (1992)

8. Huang, F.L.: Characteristic condition for exponential stability of linear dynamical systems in Hilbert spaces. Ann. Differ. Equations 1, 43-56 (1985)

9. Keddi, A.; Apalara, T.; Messaoudi, S.: Exponential and polynomial decay in a thermoelastic-Bresse system with second sound. Appl. Math. Optim. https://doi.org/10.1007/s00245-016-9376-y (to appear)

10. Lagnese, J.E.; Leugering, G.; Schmidt, J.P.: Modelling of dynamic networks of thin thermoelastic beams. Math. Methods Appl. Sci. 16, 327-358 (1993)

11. Lagnese, J.E.; Leugering, G.; Schmidt, E.J.P.G.: Modelling analysis and control of dynamic elastic multi-link structures. Systems \& Control: Foundations \& Applications. Birkhäuser Basel, Boston, MA (1994). https://doi.org/10.1007/978-14612-0273-8

12. Liu, Z.; Rao, B.: Characterization of polymomial decay rate for the solution of linear evolution equation. Z. Angew. Math. Phys. 56, 630-644 (2005)

13. Liu, Z.; Rao, B.: Energy decay rate of the thermoelastic Bresse system. Z. Angew. Math. Phys. 60, 54-69 (2009)

14. Liu, Z.; Zheng, S.: Semigroups Associated with Dissipative Systems, 398 Research Notes in Mathematics. Chapman and Hall/CRC, London (1999)

15. Najdi, N.; Wehbe, A.: Weakly locally thermal stabilization of Bresse systems. Electron. J. Differ. Equations 2014, 1-19 (2014)

16. Pazy, A.: Semigroups of Linear Operators and Applications to Partial Differential Equations. Springer, New York (1983)

17. Pruss, J.: On the spectrum of $C_{0}$ semigroups. Trans. Am. Math. Soc. 284, 847-857 (1984)

Publisher's Note Springer Nature remains neutral with regard to jurisdictional claims in published maps and institutional affiliations. 